\title{
Formation of galaxies in $\Lambda$ cold dark matter cosmologies - I. The fine structure of disc galaxies
}

\author{
M. Doménech-Moral, ${ }^{1 \star}$ F. J. Martínez-Serrano, ${ }^{1}$ R. Domínguez-Tenreiro ${ }^{2}$ \\ and A. Serna ${ }^{1}$ \\ ${ }^{1}$ Departamento de Física y Arquitectura de Computadores, Universidad Miguel Hernández de Elche, 03202-Elche, Spain \\ ${ }^{2}$ Departamento de Física Teórica, Universidad Autónoma de Madrid, Madrid, Spain
}

Accepted 2012 January 2. Received 2011 December 21; in original form 2011 May 12

\begin{abstract}
In this work we present a detailed analysis of the global and fine structure of four middlemass disc galaxies obtained from hydrodynamic simulations in a $\Lambda$ cold dark matter $(\Lambda \mathrm{CDM})$ scenario. These objects have photometric disc-to-total ratios in good agreement with those observed for late-type spirals, as well as kinematic properties in agreement with the observational I-band Tully-Fisher relation. We identify the different dynamical components at redshift zero on the basis of both orbital parameters and the binding energy of stars in the galaxy. In this way, we recognize a slowly rotating centrally concentrated spheroid, and two disc components supported by rotation: a thin disc with stars in nearly circular orbits, and a thick disc with orbital parameters transitional between the thin disc and the spheroid. The spheroidal component is composed mainly by old, metal-poor and $\alpha$-enhanced stars. The distribution of metals in this component shows, however, a clear bimodality with a low-metallicity peak, which could be related to a classical bulge formed from rapid collapse at early times, and a high-metallicity peak, which could be related to a pseudo-bulge formed from instabilities of the inner disc. The thin disc appears in our simulations as the youngest and most metal-rich component, with median stellar ages ranging from 3.8 to $6.7 \mathrm{Gyr}$. The radial distribution of ages and colours in this component is $U$-shaped: the new stars are forming in the inner regions, where the galaxy is bluer, and then migrate through secular processes reaching the outer parts. Finally, we also find in all simulated galaxies a thick disc containing about 16 per cent of the total stellar mass and with properties that are intermediate between those of the thin disc and the spheroid. Its low-metallicity stars are $\alpha$-enhanced when compared to thin disc stars of the same metallicity. The structural parameters (e.g. the scale height) of the simulated thick discs suggest that such a component could result from the combination of different thickening mechanisms that include merger-driven processes, but also long-lived internal perturbations of the thin disc.
\end{abstract}

Key words: hydrodynamics - galaxies: formation - galaxies: star formation - galaxies: structure - cosmology: theory.

\section{INTRODUCTION}

Spiral galaxies have long been recognized to contain two main stellar components: a dynamically hot spheroidal component composed by old and metal-poor stars, and a cold disc of young and more metal-rich stars.

In the past three decades, a wide range of observations has revealed the existence in almost all resolved spiral galaxies of yet another component, a thick stellar disc. This component was originally detected in the Milky Way using star counts (Gilmore \& Reid

^E-mail: mdomenech@umh.es
1983) and as an excess red flux at large galactic latitudes in external galaxies (Dalcanton \& Bernstein 2002; Yoachim \& Dalcanton 2006). It is now known to be an ubiquitous component of spiral galaxies (Dalcanton \& Bernstein 2002; Seth, Dalcanton \& de Jong 2005), but for many years it remained unclear whether it was a truly distinct component of the Milky Way thin disc. Evidence from their distinct nature came from detailed kinematic and chemical abundance studies (e.g. Fuhrmann 1998; Soubiran, Bienaymé \& Siebert 2003; Bensby, Feltzing \& Lundström 2003; Bensby et al. 2005; Reddy, Lambert \& Allende Prieto 2006).

In general, thick discs are characterized by an exponential-fit scale height, $z_{\mathrm{h}}$, larger than the thin disc by a factor of $\sim 2$ (e.g. Dalcanton \& Bernstein 2002; Yoachim \& Dalcanton 2006). In 
our Galaxy and in NGC 891, a Milky Way analogue, the $z_{\mathrm{h}}$ value seems to be roughly constant with radius (Robin et al. 2003; Ibata, Mouhcine \& Rejkuba 2009). Relative to the galactic thin disc, thick disc stars have both larger velocity dispersions and slower net rotation (Soubiran et al. 2003). It contains 10 to 35 per cent of the total baryonic mass of a galaxy (Yoachim \& Dalcanton 2006; Collins et al. 2011), and appears to be anti-correlated with the bulge mass: galaxies with small bulges have larger thick discs. Concerning their stellar population, thick disc stars are older and more metal-poor than stars in the thin disc (e.g. Gilmore, Wyse \& Jones 1995; Fuhrmann 1998). They are also significantly enhanced in $\alpha$-elements compared to thin disc stars of comparable iron abundance (e.g. Fuhrmann 1998; Bensby et al. 2003, 2005; Reddy et al. 2006), suggesting a star formation (SF) time-scale shorter than $1 \mathrm{Gyr}$ (Fuhrmann 1998; Ruchti et al. 2010).

Several formation mechanisms have been proposed in the literature to explain the origin of the different galactic components. In the prevailing picture of galaxy formation, haloes of dark matter (DM) acquire angular momentum via tidal torques from interacting structures. As gas cools and condenses into their central parts, star-forming galaxies appear with a thin disc structure (Fall \& Efstathiou 1980; White 1984). Hydrodynamical simulations, however, have shown that cold flows play a fundamental role in the building up of discs in low-mass haloes (e.g. Kereš et al. 2005; Dekel \& Birnboim 2006). The cold gas flows rapidly to the centre of galaxies from filamentary structures around haloes, where it may be swiftly accreted into the central galaxy. The lower initial gas temperature leads to shorter cooling times and to the growth of stellar discs at higher redshifts than predicted by the standard model (Brooks et al. 2009).

Within these pictures, two main formation scenarios have been proposed for the bulges of spiral galaxies (see Kormendy \& Kennicutt 2004, for a review). In classical bulges, most stars are formed during the early phase of intensive SF following collapse of the proto-galaxy and subsequent mergers. In another scenario, boxy or pseudo-bulges can also be developed through dynamical instability of the inner disc.

Thin disc instabilities are also considered as the main mechanism responsible for the thick disc formation. In most of the proposed scenarios the thick disc stars are formed in the thin disc and are dynamically heated through either the accretion of galaxy satellites (Quinn, Hernquist \& Fullagar 1993; Walker, Mihos \& Hernquist 1996; Villalobos \& Helmi 2008) or internal perturbations of the thin disc from long-lived mechanisms (e.g. the stellar scattering via resonant interactions with spiral arms; Roškar et al. 2008; Schönrich \& Binney 2009a,b) or short-lived events (e.g. perturbations due to the thin disc clumpy stellar structure at high redshifts; Bournaud, Elmegreen \& Martig 2009). In other category of models, the thick disc stars are assumed to be formed either in situ during/after gasrich mergers (Brook et al. 2004, 2005), or in external satellites that are disrupted and incorporated into the thick disc (Abadi et al. 2003b).

Numerical hydrodynamic simulations have been recognized as a powerful tool to study galaxy formation and evolution in its full cosmological context. The main difficulty in reproducing realistic disc-dominated galaxies is the transfer of gas angular momentum to the DM (Navarro \& White 1994), which results in small simulated discs with too high bulge-to-disc ratios. This catastrophic loss of angular momentum can be prevented by modelling processes [e.g. the feedback from supernova ( $\mathrm{SN})$ explosions] that regulate $\mathrm{SF}$ and the rapid consumption of gas at early times. Although the observed large fraction of late-type galaxies is still far from be- ing reproduced in simulations (Weinzirl et al. 2009), in the past decade some simulations have been able to reproduce ensemble properties of disc galaxies with gaseous discs at lower resolutions (Domínguez-Tenreiro, Tissera \& Sáiz 1998; Sáiz et al. 2001), as well as to produce individual examples of realistic disc galaxies in $\Lambda$ cold dark matter $(\Lambda \mathrm{CDM})$ universes (e.g. Abadi et al. 2003b; Sommer-Larsen, Götz \& Portinari 2003; Brook et al. 2004; Governato et al. 2007, 2009; Scannapieco et al. 2008). These simulations have generated extended stellar disc components with high specific angular momentum and with a significant fraction of young stars (Scannapieco et al. 2008). Although an old and significant spheroid is almost always present (Abadi et al. 2003a; SommerLarsen et al. 2003; Governato et al. 2007; Scannapieco et al. 2008), these discs show realistic exponential scale lengths and integrated colours. Moreover, they fall on the region defined by the observational $I$-band Tully-Fisher relation and have bulge-to-disc ratios or stellar mass-to-light ratios similar to observational counterparts. The presence of a thick disc component is evidenced in some simulated discs by both a double exponential vertical structure and a velocity dispersion versus age relation that resemble those observed in the Milky Way (Abadi et al. 2003b; Brook et al. 2004).

The aim of this and forthcoming papers is to study the formation mechanisms of the different galactic components (bulge, halo, thin disc and thick disc) by means of cosmo-hydrodynamic simulations. For this purpose we follow the evolution of four different disc galaxies in a $\Lambda \mathrm{CDM}$ cosmology, using an OPENMP parallel version of the DEVA code (Serna, Domínguez-Tenreiro \& Sáiz 2003) which includes the chemical feedback and cooling methods described in Martínez-Serrano et al. (2008). The present work is focused on their global and fine structure at $z=0$ and their comparison with observations. We will show in this paper that the dynamical and chemical properties of these simulated galaxies have values consistent with observations and contain valuable information about their formation processes.

This paper is organized as follows. In Section 2 we briefly describe the simulation code and the main characteristics of our simulations. In Section 3 we present the general properties of our simulated galaxies at $z=0$ and compare with observational disc properties. In Section 4 we identify the dynamical components in the simulated galaxies (Section 4.1), to perform a detailed description and comparison of these components with observational references. We analyse the vertical density profiles (Section 5), stellar ages (subsection 6.1), age and colour gradients (subsection 6.2) and chemical properties (Section 7) of such components. Finally, in Section 8 we summarize and discuss our main results.

\section{THE SIMULATIONS}

All simulations were performed with an OPENMP parallel version of DEVA, an AP3M+SPH code specially designed so that conservation laws (e.g. momentum, energy, angular momentum and entropy) hold as accurately as possible (see Serna et al. 2003, for details). The code also includes methods to account for subresolution physical processes such as SF, gas restitution from stars, chemical feedback and cooling. These methods are extensively described in MartínezSerrano et al. (2008). We will summarize them below.

\subsection{Star formation}

SF is implemented through a Kennicutt-Schmidt-like law:

$\frac{\mathrm{d} \rho}{\mathrm{d} t}=-\frac{c_{*} \rho}{t_{\mathrm{g}}}$ 
Table 1. Principal characteristics of simulations: mass of a single baryonic particle $\left(m_{\mathrm{bar}}\right)$, SF parameters, density threshold $\left(\rho_{*}\right)$ and efficiency $\left(c_{*}\right)$, virial radius $\left(r_{\mathrm{vir}}\right)$, spin parameter of the DM halo $\left(\lambda_{\mathrm{DM}}\right)$, the total $\left(M_{\mathrm{vir}}\right)$, stellar $\left(M_{\mathrm{star}}^{\mathrm{vir}}\right)$ and gas $\left(M_{\mathrm{gas}}^{\mathrm{vir}}\right)$ masses inside the virial radius.

\begin{tabular}{lcccccccc}
\hline Galaxy & $\begin{array}{c}m_{\text {bar }} \\
\left(\mathrm{M}_{\odot}\right)\end{array}$ & $\begin{array}{c}\rho_{*} \\
\left(\mathrm{~g} \mathrm{~cm}^{-3}\right)\end{array}$ & $c_{*}$ & $\begin{array}{c}r_{\mathrm{vir}} \\
\left(h^{-1} \mathrm{kpc}\right)\end{array}$ & $\lambda_{\mathrm{DM}}$ & $\begin{array}{c}M_{\text {vir }} \\
\left(10^{11} \mathrm{M}_{\odot}\right)\end{array}$ & $\begin{array}{c}M_{\text {star }}^{\text {vir }} \\
\left(10^{10} \mathrm{M}_{\odot}\right)\end{array}$ & $\begin{array}{c}M_{\mathrm{gas}}^{\mathrm{vir}} \\
\left(10^{10} \mathrm{M}_{\odot}\right)\end{array}$ \\
\hline LD-5003A & $3.82 \times 10^{5}$ & $6 \times 10^{-26}$ & 0.010 & 106.12 & 0.019720 & 1.95 & 1.79 & 0.63 \\
LD-5101A & $3.79 \times 10^{5}$ & $6 \times 10^{-26}$ & 0.010 & 90.55 & 0.034233 & 1.21 & 1.49 & 0.48 \\
HD-5004A & $3.94 \times 10^{5}$ & $6 \times 10^{-26}$ & 0.010 & 127.00 & 0.023273 & 3.35 & 3.74 & 1.02 \\
HD-5103A & $3.78 \times 10^{5}$ & $6 \times 10^{-26}$ & 0.010 & 141.62 & 0.098032 & 4.66 & 3.42 & 0.88 \\
HD-5004B & $3.94 \times 10^{5}$ & $1 \times 10^{-25}$ & 0.008 & 128.10 & 0.023387 & 3.44 & 3.57 & 1.31 \\
HD-5103B & $3.78 \times 10^{5}$ & $1 \times 10^{-25}$ & 0.008 & 140.53 & 0.096513 & 4.55 & 3.36 & 0.89 \\
HD-5004L & $3.12 \times 10^{6}$ & $3 \times 10^{-26}$ & 0.010 & 128.47 & 0.027249 & 3.48 & 3.87 & 0.96 \\
\hline
\end{tabular}

where $\rho$ is the gas density, $c_{*}$ is a dimensionless SF efficiency parameter, and $t_{\mathrm{g}}$ is a characteristic time-scale chosen to be equal to the maximum of the local dynamical time and the local cooling time. We consider that gas particles are eligible to form stars if they are located in a region with a convergent flow and a gas density higher than a density threshold $\rho_{*}$. At each time-step we compute the probability that those eligible gas particles form stars in the considered time interval, and draw random numbers to decide which of them will form stars. Then, each of these randomly selected gas particles is transformed into one stellar particle, which is assumed to be a single stellar population (SSP) of a given age and mass (see Table 1). In the simulations presented in this paper, stellar masses within each SSP were assumed to be distributed according to the Salpeter initial mass function (IMF) (Salpeter 1955), with a mass range of $\left[M_{l}, M_{u}\right]=[0.1,100] \mathrm{M}_{\odot}$.

Since our cosmological simulations do not resolve star-forming molecular clouds, in the above scheme the choice of the $\rho_{*}$ value is based on the fact that the ISM can be represented by a lognormal density probability distribution function (PDF) (see e.g. Kravtsov 2003; Wada \& Norman 2007). The amount of gas eligible for SF is represented by the high-density (HD) part of the PDF, which in turn is a function of the total galaxy gas mass and turbulence. The choice of the $\rho_{*}$ value must allow for the HD star-forming part of the PDF to be well resolved or, at least, to contain a converged amount of star-forming mass for the adopted numerical resolution (Agertz, Teyssier \& Moore 2011). Despite this theoretical dependence of the SF parameters on the local properties, and probably also on redshift, for a given simulation such parameters are usually taken as constants.

Discrete energy injection processes have been found to play an important role in the self-regulation of the SF processes in galaxies. Positive feedback effects can help to halt the overproduction of stellar mass and, when implemented in simulations, help to properly reproduce some properties of disc galaxies. The most widely employed model in cosmological hydrodynamical simulations assumes a strong explicit feedback combined with a high SF efficiency (e.g. Scannapieco et al. 2008, 2011a; but see also the discussion in Kereš et al. 2009). However, a number of authors (see Agertz et al. 2011 , for a recent work) have shown that the sub-parsec processes of SF self-regulation in simulated disc galaxies can also be implicitly modelled by means of a low SF efficiency. Since our aim in this paper is to test the minimal conditions for the formation of realistic discs, we have adopted the latter model for subresolution physics. According with this approach, we have chosen inefficient SF parameters (see Table 1), which implicitly account for the regulation of SF by discrete energy injection processes.

\subsection{Chemical feedback and cooling}

Methods for chemical feedback and cooling are also those described in Martínez-Serrano et al. (2008). These methods account for the full dependence of radiative cooling on the detailed composition of the gas, through a fast algorithm based on a metallicity parameter, $\zeta(T)$, which takes into account the weight of the different elements on the total cooling function. Such a method for cooling is important to prevent an overestimation of the metallicity-dependent cooling rate.

The release to the ISM of newly produced elements, as byproducts of stellar evolution and death, is included in our code through a stochastic algorithm that completely removes the assumption of instantaneous recycling of stellar ejections. In this algorithm, the metal ejection from stars is progressively released to the ISM as increments in the metal content of nearby gas particles. The delayed gas restitution from stars is also taken into account by means of a probabilistic approach that reduces the statistical noise and, hence, that allows for the study of the inner chemical structure of objects with moderately high numbers of particles. Furthermore, once the stellar production of metals has been released to the ISM, the chemical elements are distributed and mixed through the gas component by means of a diffusion law that models, without the explicit inclusion of stellar winds, the subresolution turbulent mixing of metals.

In the computation of the metal production, our code accounts for the full dependence on the detailed chemical composition of stellar particles by means of the $Q_{i j}$ formalism (Talbot \& Arnett 1973), which relates each nucleosynthetic product to its sources. Such a $Q_{i j}$ formalism is important to prevent a significant underestimation of the $[\alpha / \mathrm{Fe}]$ ratio in simulated galaxies. In this paper, we have used the stellar evolution data described in Gavilán, Buell \& Mollá (2005) for low- and intermediate-mass stars, and Woosley \& Weaver (1995) for high-mass stars. SNIa rates were computed according to Ruiz-Lapuente et al. (2000) and their element production according to Iwamoto et al. (1999). We have considered the evolution of the following elements: $\mathrm{H},{ }^{4} \mathrm{He},{ }^{12} \mathrm{C},{ }^{13} \mathrm{C},{ }^{14} \mathrm{~N},{ }^{16} \mathrm{O},{ }^{20} \mathrm{Ne},{ }^{24} \mathrm{Mg},{ }^{28} \mathrm{Si}$, ${ }^{32} \mathrm{~S},{ }^{40} \mathrm{Ca}$ and ${ }^{56} \mathrm{Fe}$.

\subsection{Simulated disc galaxies}

The simulation of each disc galaxy consists of a cosmological zoomin that includes high-resolution gas and DM for the flow converging region that generates the main object. The rest of the simulation box is sampled by low-resolution DM particles that account for tidal forces over the flow converging region. 
LD-5003A
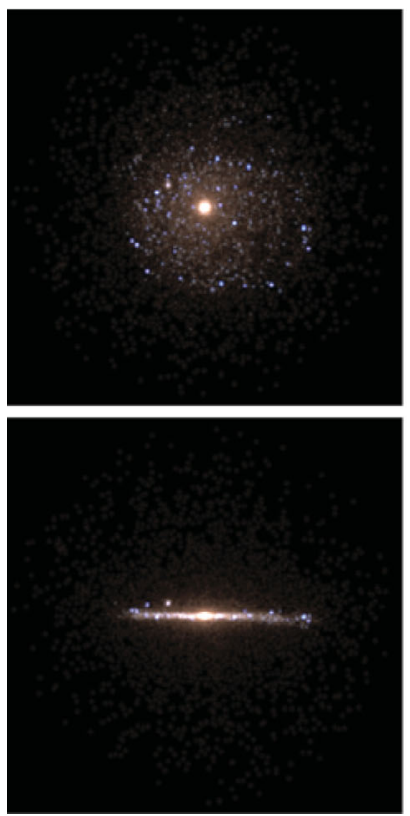

LD-5101A
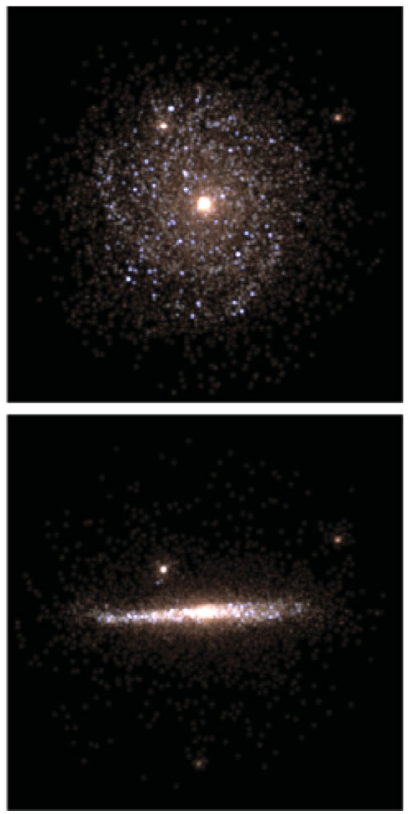

HD-5004A
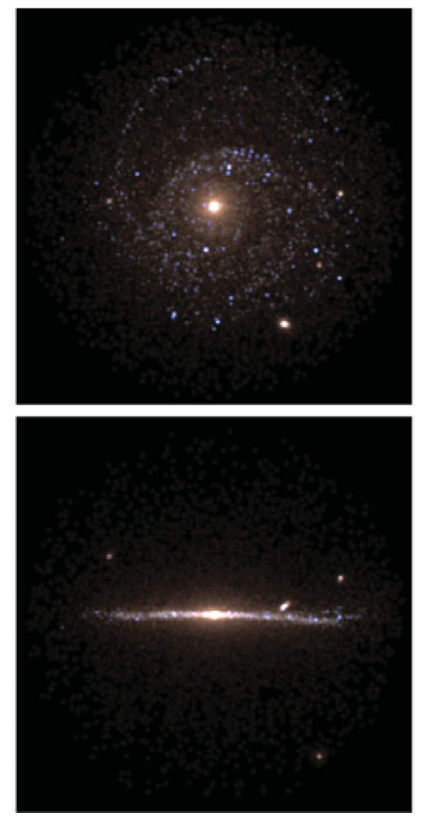

HD-5103A
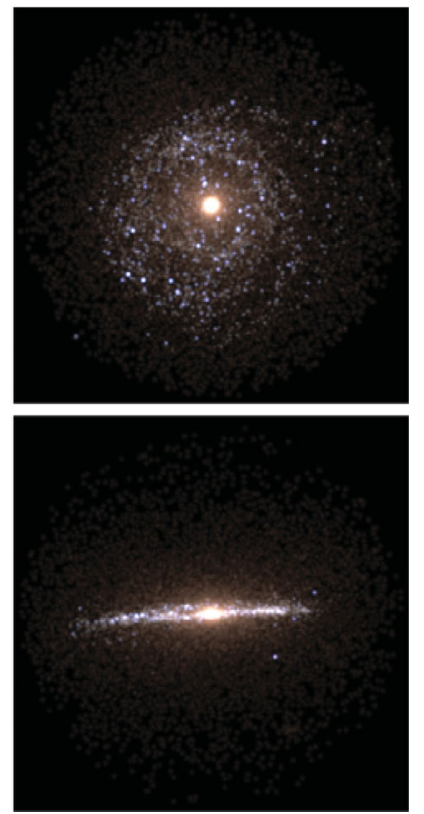

Figure 1. Face-on and edge-on synthetic images obtained using Bruzual \& Charlot (2003) models at $z=0$. All images are $40 \mathrm{kpc}$ side.

As a first step, we performed two low-resolution simulations that consisted of two different Monte Carlo realizations of the same cosmological model: a flat $\Lambda \mathrm{CDM}$ universe (with $\Omega_{\Lambda}=0.723$, $\Omega_{\mathrm{m}}=0.277, \Omega_{\mathrm{b}}=0.04, \sigma_{8}=0.811$ and $\left.h=0.7\right)$ within a periodic box of $10 \mathrm{Mpc}$ per side. ${ }^{1}$

From each of these low-resolution simulations, we selected two gas-rich objects with a prominent gas disc at $z=0$ not apparently perturbed from recent major mergers: the most massive one from a sparsely populated region and the most massive one from a crowded region. These selection criteria then focus on massive regular spiral galaxies extracted from both low- and HD regions and, hence, with different histories of mergers and accretion events. For each selected object, we traced back the particles inside its virial radius until the initial redshift $z_{\text {init }}=31$. We then computed the convex hull (Barber, Dobkin \& Huhdanpaa 1996) enclosing these particles at $z_{\text {init }}$ and substituted all the particles inside the convex hull by their high-resolution counterparts. Gas particles outside the hull were eliminated and their masses added to the low-resolution DM component, thus obtaining the initial conditions for each highresolution simulation. The galaxies obtained in this way have a full history of mergers and accretion events in a cosmological context, without any assumptions made for their initial conditions beyond the cosmology and the initial conditions generator used (Prunet et al. 2008). Table 1 summarizes the main characteristics of each high-resolution simulation, with a gravitational softening of $\epsilon_{\mathrm{g}}=$ $400 \mathrm{~h}^{-1} \mathrm{pc}$ and a minimum hydrodynamical smoothing length half this value. In this table, LD-5003A and LD-5101A correspond to objects selected in low-density (LD) regions, while HD-5004A and HD-5103A correspond to objects from HD regions. All these objects (labelled as A) were simulated with identical SF parameters.

\footnotetext{
${ }^{1}$ Although this box side implies a lack of very massive objects and galaxy groups, it has been proved that it has little effect on the internal properties of the haloes (Power \& Knebe 2006) and on the formation of individual galaxies.
}

The two objects selected from crowded regions experienced major mergers at moderately low redshifts. In principle, the ability of their discs to regenerate after their last major merger, as well as the resulting properties of their fine structure, could be sensitive to the choice for SF efficiency. In order to test the possible effects of SF efficiency, these two objects were re-simulated with less efficient SF parameters (simulations labelled as B: HD-5004B and HD-5103B).

Table 1 also shows the total, stellar and gas masses enclosed by the virial radius of each object. We can see from this table that our simulated galaxies have virial masses in the range 1.2 to $4.7 \times$ $10^{11} \mathrm{M}_{\odot}$ and stellar masses of $\sim 10^{10.5}$, placing them among middlemass spiral galaxies. Therefore, they are less massive than the Milky Way (with virial mass of $\sim 1.2 \times 10^{12} \mathrm{M}_{\odot}$ ) and their masses are comparable to the least massive simulated galaxies presented in recent works (e.g. Governato et al. 2007; Scannapieco et al. 2009; Guedes et al. 2011).

\section{GENERAL PROPERTIES OF DISCS}

\subsection{Bulge-disc decomposition: $D / T$ ratios}

Fig. 1 shows the face-on and edge-on synthetic images of the four main simulated objects at $z=0$, where SSP ages, masses and metallicities have been converted into luminosities using the Bruzual \& Charlot (2003) models. ${ }^{2}$ In all these images, a conspicuous disc component can be appreciated even for the object HD-5103A, where the disc structure has survived a recent major merger at $z \sim 0.3$. Although the synthetic image of HD-5103A does not show clear evidence of such a merger, we will see in Section 4 that this event has still a strong imprint in several physical properties.

In order to quantify the importance of each component, Fig. 2 shows the face-on $r$-band luminosity profiles as well as the fits

\footnotetext{
${ }^{2}$ Dust effects have not been considered in this work, so, in order to minimize these effects, photometric studies have been done with galaxies seen face-on.
} 


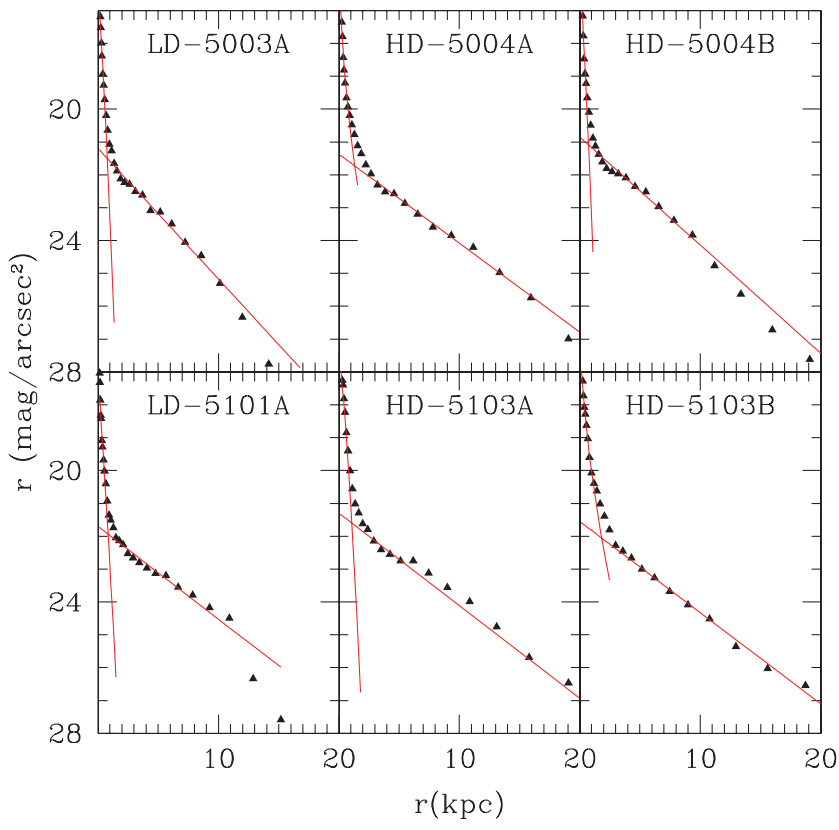

Figure 2. Face-on luminosity profiles in the $r$ band (filled triangles) and their fits obtained with the bulge-disc decomposition (red solid lines).

obtained from their bulge-disc decomposition (see Table 2 for the fitting parameters in different bands). In each band we have performed a one-dimensional (1D) fit which combines a Sérsic law for the inner region and a simple exponential profile in the outer parts. This fit provides a very good description of the stellar luminosity profile up to a limit of $\sim 24 \mathrm{mag} \operatorname{arcsec}^{-2}$. In the outer regions, where a second exponential should be employed (see Martínez-Serrano et al. 2009), some deviation is instead observed. Such a deviation has, however, little effect on the global fit quality measured with the mean square deviation, $\chi^{2}$.

Recent works have used 2D fitting packages developed for use in observational studies (e.g. Gadotti 2008) to perform the bulge-disc decomposition. They have shown that different results can be obtained when different fitting techniques are employed, and also that the effects of neglecting bars when they are present can alter the resulting bulge-to-total and disc-to-total ratios. In order to test the effects of such different techniques, we have also performed fits to face-on synthetic images using GALFIT (Peng et al. 2010). The resulting fits show similar values for the scale length and effective radius, somewhat higher values for the Sérsic shape parameter $n$ and minor-to-major ratios very close to one. The resulting $D / T$ ratios were roughly the same as those obtained from 1D fits, but with a higher uncertainty due to the additional parameters (point spread function, dust processing,...) required to generate the synthetic images. Due to the similitude of results and the fact that our objects have a high azimuthal symmetry, we have chosen to show here only the one-dimensional bulge-disc decompositions.

The $D / T$ ratios, measured in our objects from their 1D fits in different photometric bands, are shown in Table 2. In general, they are in good agreement with those expected for spiral galaxies (e.g. Laurikainen et al. 2007; Oohama et al. 2009) and are comparable with those recently obtained in simulations (e.g. Governato et al. 2009; Guedes et al. 2011). Further, the values obtained from Sérsic fits to the total light distribution are also in good correspondence with both the luminosity-size and stellar mass-size relations for disc galaxies given by Shen et al. (2003). In order to compare our results with the compilation reported by Graham \& Worley (2008),

Table 2. Face-on photometric decomposition of simulated galaxies in different bands. $r_{\mathrm{e}}$ stands for the effective radius of the bulge, $r_{\mathrm{s}}$ for the scale length of the disc. $L_{T}$ and $L_{D}$ represent the luminosity in the central region and the luminosity of the disc. We also show the $\chi^{2}$ value of the fit. $D / T^{P}$ is defined as the disc-to-total ratio obtained using the photometric decomposition, and $\mathrm{M}$ as the absolute magnitude in each band.

\begin{tabular}{|c|c|c|c|c|c|c|c|c|c|}
\hline Galaxy & Band & $n$ & $\begin{array}{c}r_{\mathrm{e}} \\
(\mathrm{kpc})\end{array}$ & $\begin{array}{c}r_{\mathrm{s}} \\
(\mathrm{kpc})\end{array}$ & $\begin{array}{c}L_{T} \\
\left(\mathrm{mag} \mathrm{arcsec}^{-2}\right)\end{array}$ & $\begin{array}{c}L_{D} \\
\left(\mathrm{mag}_{\operatorname{arcsec}}{ }^{-2}\right)\end{array}$ & $\begin{aligned} & \chi^{2} \\
& \times 10^{-5}\end{aligned}$ & $D / T^{P}$ & $\begin{array}{c}\mathrm{M} \\
(\mathrm{mag})\end{array}$ \\
\hline \multirow{3}{*}{ LD-5003A } & $r$ & 0.78 & 0.28 & 2.72 & 18.0 & 17.0 & 6.0 & 0.72 & -20.20 \\
\hline & $g$ & 0.63 & 0.27 & 2.86 & 18.7 & 17.5 & 9.7 & 0.75 & -19.69 \\
\hline & $i$ & 0.83 & 0.29 & 2.67 & 17.7 & 16.8 & 5.3 & 0.70 & -20.48 \\
\hline \multirow{3}{*}{ LD-5101A } & $r$ & 0.90 & 0.32 & 3.83 & 18.6 & 16.8 & 14.3 & 0.84 & -20.03 \\
\hline & $g$ & 0.73 & 0.31 & 4.23 & 19.2 & 17.1 & 21.3 & 0.87 & -19.58 \\
\hline & $i$ & 0.96 & 0.32 & 3.76 & 18.3 & 16.6 & 12.0 & 0.83 & -20.28 \\
\hline \multirow{3}{*}{ HD-5004A } & $r$ & 2.21 & 0.37 & 4.00 & 17.3 & 16.4 & 7.7 & 0.70 & -20.83 \\
\hline & $g$ & 1.70 & 0.33 & 4.05 & 18.0 & 16.8 & 7.3 & 0.76 & -20.29 \\
\hline & $i$ & 2.38 & 0.39 & 3.98 & 16.9 & 16.1 & 7.7 & 0.68 & -21.12 \\
\hline \multirow{3}{*}{ HD-5103A } & $r$ & 0.73 & 0.39 & 3.85 & 17.3 & 16.4 & 5.1 & 0.69 & -20.77 \\
\hline & $g$ & 0.58 & 0.37 & 3.83 & 18.0 & 16.8 & 9.1 & 0.75 & -20.26 \\
\hline & $i$ & 0.78 & 0.40 & 3.85 & 16.9 & 16.1 & 5.2 & 0.68 & -21.05 \\
\hline \multirow{3}{*}{ HD-5004B } & $r$ & 0.80 & 0.26 & 3.29 & 17.6 & 16.2 & 9.0 & 0.77 & -20.88 \\
\hline & $g$ & 0.58 & 0.25 & 3.47 & 18.3 & 16.7 & 17.0 & 0.81 & -20.37 \\
\hline & $i$ & 0.88 & 0.26 & 3.21 & 17.3 & 16.0 & 7.3 & 0.76 & -21.16 \\
\hline \multirow{3}{*}{ HD-5103B } & $r$ & 1.75 & 0.55 & 3.90 & 17.0 & 16.6 & 6.9 & 0.58 & -20.77 \\
\hline & $g$ & 1.63 & 0.48 & 3.65 & 17.6 & 17.0 & 6.4 & 0.64 & -20.24 \\
\hline & $i$ & 1.76 & 0.57 & 3.99 & 16.6 & 16.3 & 7.3 & 0.56 & -21.05 \\
\hline \multirow{3}{*}{ HD-5004L } & $r$ & 1.01 & 0.31 & 3.76 & 17.3 & 16.2 & 5.0 & 0.72 & -20.87 \\
\hline & $g$ & 1.07 & 0.30 & 4.02 & 17.9 & 16.8 & 6.0 & 0.73 & -20.31 \\
\hline & $i$ & 0.98 & 0.31 & 3.67 & 17.0 & 16.0 & 5.0 & 0.72 & -21.17 \\
\hline
\end{tabular}


we have also computed the bulge-disc decomposition in the $K$ band. The median values obtained for the shape parameter $(n=1.46)$, the effective radius $\left(r_{\mathrm{e}}=0.37 \mathrm{kpc}\right)$ and the scale length $\left(r_{\mathrm{s}}=3.71 \mathrm{kpc}\right)$ of our discs are in good agreement with those reported for spiral galaxies.

Although these results must be taken with caution, due to uncertainties introduced by different fitting (Gadotti 2008) and decomposition (e.g. Abadi et al. 2003b; Scannapieco et al. 2010, see also the Section 4.1 below) methods, the relatively small size of bulges obtained in these simulations is an important result by itself, given the usual problems in reproducing realistic disc galaxies with cosmological simulations (Abadi et al. 2003a; Burkert 2009). As already quoted in Section 1, the main problem hindering the formation of large galactic discs is the loss of gas angular momentum at early stages of galaxy formation, which results in objects with too high bulge-to-disc ratios. This catastrophic loss of gas angular momentum can be prevented by including feedback processes avoiding the early in-fall of gas and its rapid consumption (e.g. Sommer-Larsen et al. 2003; Governato et al. 2007; Scannapieco et al. 2008; Agertz et al. 2011). The cosmological simulations reported in this work, where energy feedback is implicitly included in the SF parameters, lead to disc galaxies with a bulge component comparable to those observed in Sab-Sb galaxies (Oohama et al. 2009).

\subsection{Rotation curves and the Tully-Fisher relation}

Fig. 3 shows the circular velocity curves for the DM and the baryonic components (dashed and dotted lines, respectively) of the simulated galaxies, computed as

$V_{i}^{2}(r)=\frac{G M_{i}(<r) r^{2}}{\left(r^{2}+\epsilon_{\mathrm{g}}^{2}\right)^{3 / 2}}$

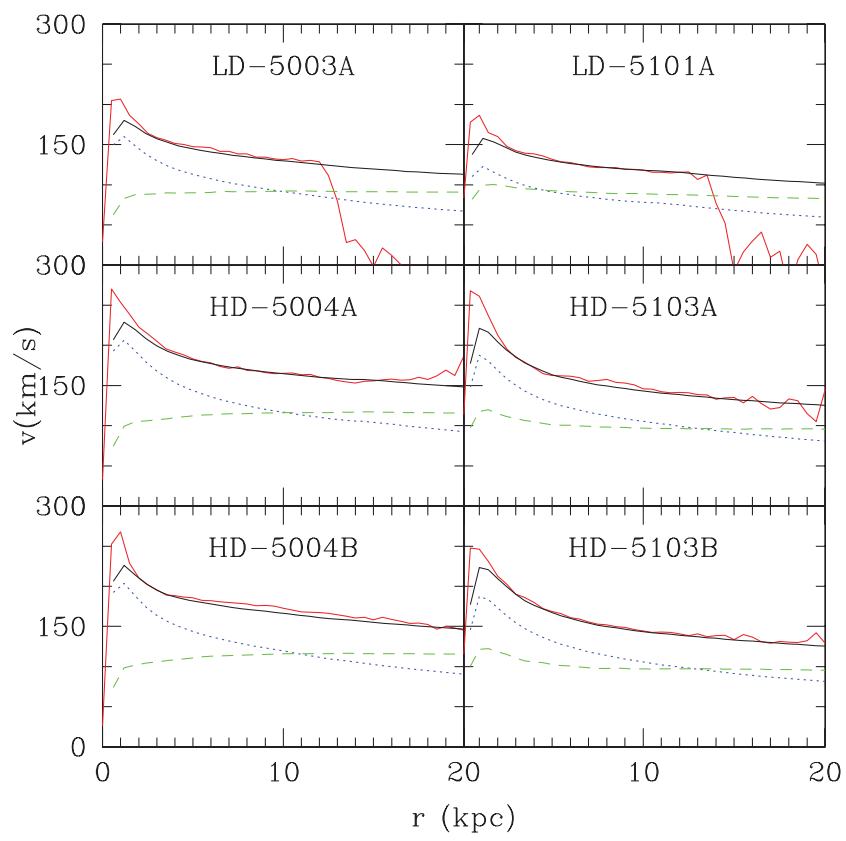

Figure 3. Circular velocity profiles of the simulated galaxies. Black solid line stands for the total mass distribution $\left(V_{\text {circ }}\right)$, as well as green dashed and blue dotted lines for the DM $\left(V_{\mathrm{DM}}\right)$ and baryonic $\left(V_{\mathrm{bar}}\right)$ contributions, respectively. The rotational mean velocities for the gas particles are displayed with a red solid line.

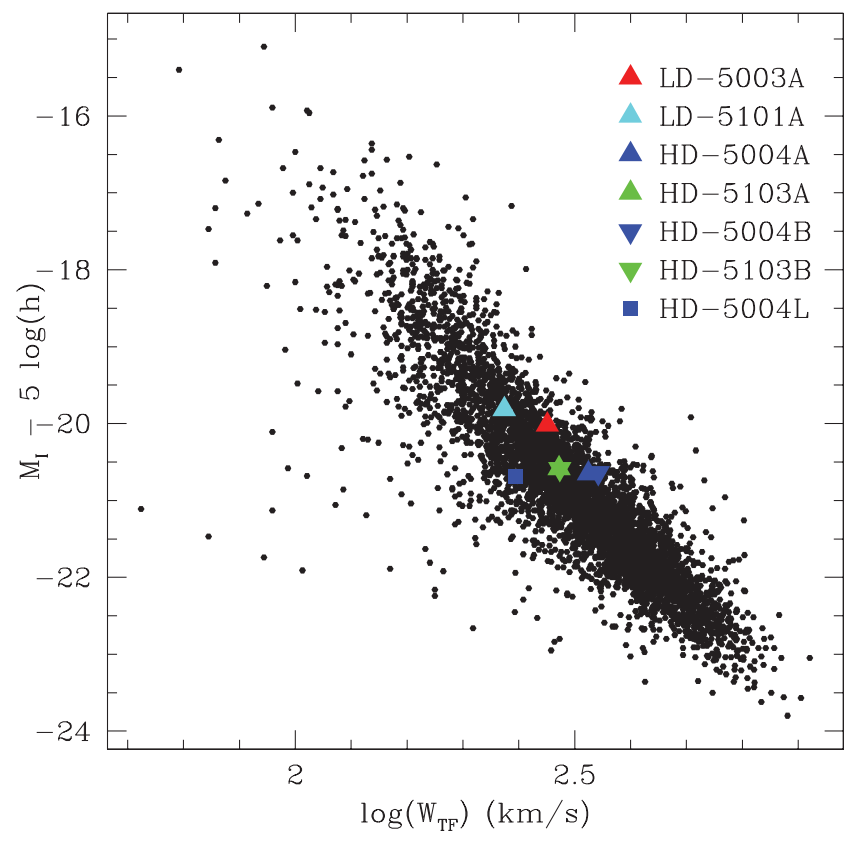

Figure 4. Black dots correspond to the $I$-band Tully-Fisher relation from the catalogue of Springob et al. (2007). Coloured triangles represent our simulated galaxies. The circular velocity corresponds to that measured at $R=2.2 r_{\mathrm{s}}$, and magnitudes have been computed using the Bruzual \& Charlot (2003) models.

with $i=$ baryons (bar) and DM. Solid lines are the circular velocities, $V_{\text {circ }}^{2}(r)=V_{\mathrm{DM}}^{2}(r)+V_{\mathrm{bar}}^{2}(r)$, where $r$ stands for the radial distance to the galactic centre. As it can be clearly seen from this figure, the baryonic component of the galaxy dominates the dynamical properties in the innermost region, while the contribution of DM is approximately constant across the galaxy, dominating the curve in the external region. The red solid line stands for the rotational velocity measured from the gas particle movement. We can see that gas particles closely follow the circular velocity curve of the objects, except in the innermost region where they are too peaky. This is a common problem in cosmological simulations (e.g. Abadi et al. 2003a; Governato et al. 2007; Stinson et al. 2010; Agertz et al. 2011; and less severe in Scannapieco et al. 2011b), in which differences between the peak circular velocity and the velocity at large radii are larger than in most observed galaxies (Catinella, Giovanelli \& Haynes 2006).

In Fig. 4 we show the $I$-band Tully-Fisher relation for our objects (coloured triangles) as well as for the sample of spiral galaxies from the catalogue of Springob et al. (2007). All the simulated objects lie within the region defined by the observational data scatter, although with some systematic trend to be fainter than the average for spirals of similar rotation speed.

\subsection{Angular momentum}

In order to compare the angular momentum of the different components of our simulated galaxies with observations, in Fig. 5 we plot the specific angular momentum $(j=J / M)$ at $z=0$ as a function of mass $(M)$ (left-hand panel), and as a function of the rotation speed (right-hand panel). Recent works have made use of similar representations (Scannapieco et al. 2011a; Torrey et al. 2011) with the same purpose, since it provides a straightforward comparison with observations and can help to pinpoint any angular momentum problems. In the first plot, the specific angular momentum and mass 

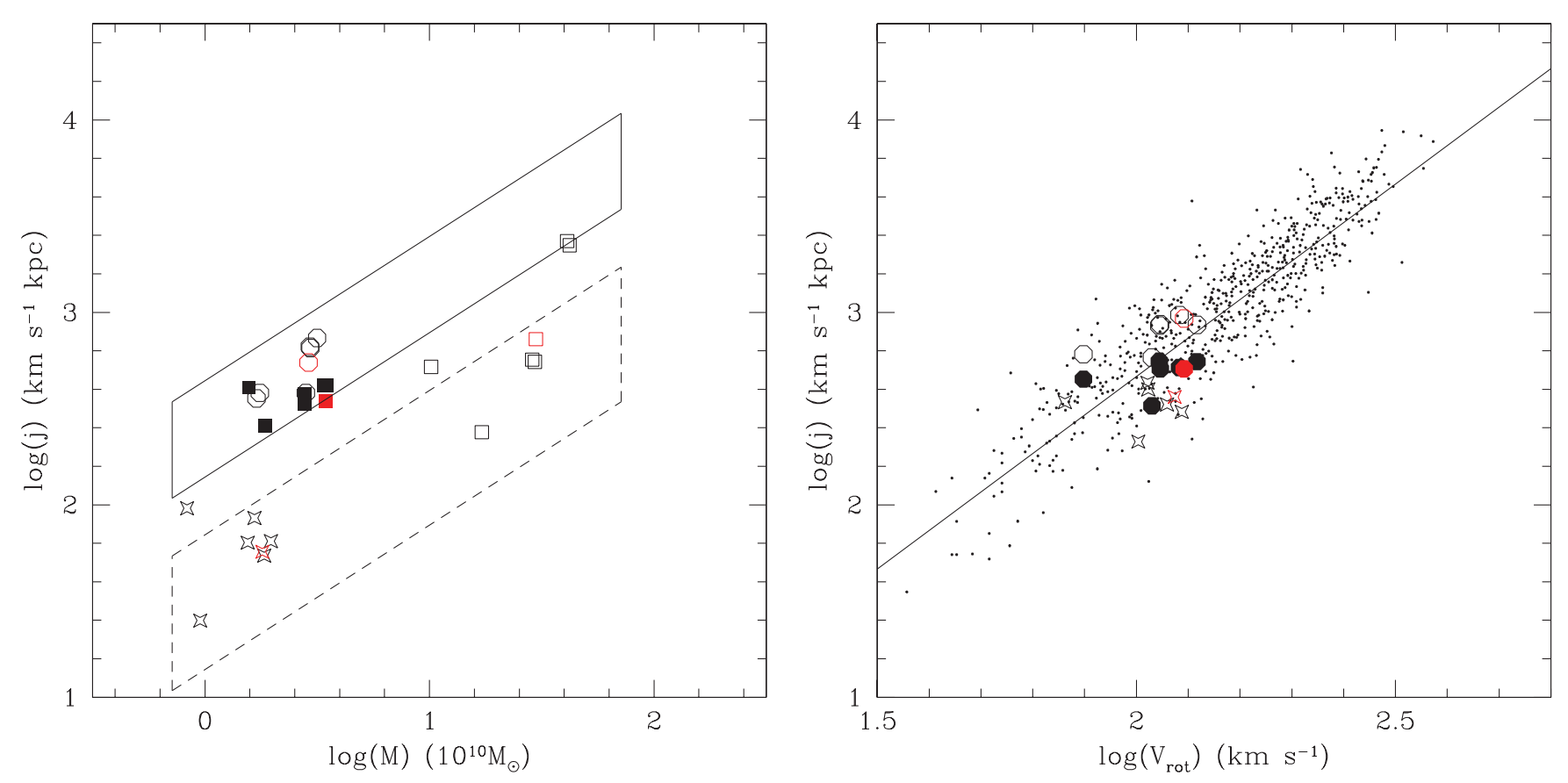

Figure 5. Left-hand panel: specific angular momentum at $z=0$ versus the mass for the DM haloes (open squares), baryonic discs (filled squares) and stellar bulges (starred symbols). Open circles are computed using the same methods than for observational galaxies: the specific angular momentum is obtained from the rotational curve, and masses from the mass-luminosity relation. Red symbols correspond to the low-resolution simulation. The limit radial distances for this plot were $r_{\text {vir }}$ for DM haloes, $r_{\mathrm{opt}}=3.2 r_{\mathrm{s}}$ for discs and $r_{\mathrm{e}}$ for bulges. The solid and dashed boxes enclose the observational regions for spiral and elliptical galaxies, respectively, as given by Fall (1983). Right-hand panel: specific angular momentum versus rotation speed at $z=0$. Small black dots correspond to late-type spirals compiled by Navarro (1998). Filled circles correspond to the simulated galaxies, assuming that the rotation velocity is that measured at $r=2.2 r_{\mathrm{s}}$, and the angular momentum the sum of specific angular momentum of baryonic particles. Open circles indicate the location of the disc component, computed adopting the same angular momentum estimator as applied to observed data, $j=2 r_{\mathrm{s}} v_{\mathrm{c}}$. Starred open symbols represent angular momentum and rotation velocity of the stellar component.

of dark haloes (open squares), stellar bulges (starred symbols) and baryonic discs (filled squares) are represented separately. The mass and angular momentum of the DM haloes have been estimated using particles within the virial radius, while those of the bulges and discs have been estimated with stars ${ }^{3}$ within $r<r_{\mathrm{e}}$ and baryons within $r<r_{\text {opt }}=3.2 r_{\mathrm{s}}$ (i.e. in a purely exponential disc $r_{\mathrm{opt}}$ is the radius enclosing 83 per cent of the disc mass), respectively (Serna et al. 2003; Scannapieco \& Tissera 2003). From this plot we can see that the disc components and the dark haloes have comparable specific angular momentum, so that disc particles have collapsed conserving, on average, their angular momentum. On the contrary stellar bulges have been formed from material that had lost most of its angular momentum, or that never acquired it. In the figure we also include the boxes enclosing the observational regions covered by spirals (solid line) and ellipticals (dashed line) as given by Fall (1983).

Given that the observational estimates of specific angular momentum and mass are derived from observational parameters, we also adopt an observational-like approach, and compute the specific angular momentum and masses of our discs, applying the method of Fall (1983) directly to our simulated discs (open circles in left-hand panel of Fig. 5). The specific angular momentum is obtained from the rotation curve as $j=2 v_{\mathrm{c}} r_{\mathrm{s}}$ where $v_{\mathrm{c}}=V_{\text {circ }}\left(R_{25}\right)$ is the circular

\footnotetext{
${ }^{3}$ We will refer to stellar particles in the simulation by the generic name 'stars', even though they are actually assumed to be SSPs of a given mass (see Table 1).
}

velocity at $r_{25}$, and masses are derived assuming a constant massluminosity relation $\left(M / L_{B}\right)=3.0$. We see from this figure that all simulated discs lie inside the region defined by observed spirals and close to the values computed using observational methods, although the latter show greater values for the specific angular momentum. Similarly stellar bulges lie in the region defined by ellipticals, except for some cases that still evidence the orbital angular momentum of merging structures.

Another way to quantify any potential angular momentum problem is to represent $j$ as a function of $v_{\text {rot }}$. This approach has been followed recently by Croft et al. (2009) and Abadi et al. (2003a) among others. Navarro (1998) provides a compilation of specific angular momentum of observed discs derived using the estimator described above: $j=2 v_{\mathrm{c}} r_{\mathrm{s}}$. In the right-hand panel of Fig. 5 we plot the observed data (small dots) along with our objects using three different estimators for $j$ and $v_{\text {rot }}$. Filled circles represent the sum of specific angular momentum of baryonic particles, and their rotational velocity $v_{\text {rot }}$ is that measured at $r=2.2 r_{\mathrm{s}}$. Open circles are computed using the observational estimator for $j$, as in the right-hand panel of Fig. 5, and the same estimator for $v_{\text {rot }}$. Open starred symbols represent the specific angular momentum of star particles. Their $v_{\text {rot }}$ is derived using the rotation curve of stars only. While all points lie within the observationally defined region, it can be appreciated that points derived using the actual baryonic angular momentum (filled circles) have lower angular momentum than the observational-like estimates (open circles). This behaviour was also observed in the left-hand panel of Fig. 5, and points to the fact that observational estimates, assuming a flat rotation curve, 
can under- or overestimate the real value of the specific angular momentum, depending on the trend that the rotation curve actually has. The rotation curves of our simulated galaxies decrease with increasing radius owing to the relative importance of the bulge, as do most simulated discs in the literature. Another effect is that stellar components generally have lower angular momentum than the total baryonic component. Given that we take into account all the star particles in this estimate, including an old bulge and thick disc, this is expected, since these components are not fully supported by rotation.

\subsection{Resolution effects}

In order to test for resolution effects in our simulations, we have run another instance (HD-5004L) of the HD-5004A object, but with half the spatial resolution and a mass resolution of about eight times lower than in the high-resolution run. The gravitational softening length was scaled to $\epsilon_{\mathrm{g}}=800 \mathrm{~h}^{-1} \mathrm{pc}$, as well as the density threshold for SF, which was set to $\rho_{*}=3 \times 10^{-26} \mathrm{~g} \mathrm{~cm}^{-3}$ in order to roughly have the same overall SF efficiency as in HD-5004A (i.e. an object stellar to baryonic mass ratio of $\sim 85$ per cent). The main characteristics of this low-resolution simulation, as well as some general properties of the resulting object, are shown in the last row of Tables 1, 2 and 4.

We can see from Table 1 that the resulting virial radius and virial mass at $z=0$ are nearly the same as those of the high-resolution counterpart. Both runs give also very close values for the stellar (a difference of $\sim 4$ per cent) and gas (a difference of $\sim 7$ per cent) mass inside the virial radius.

The photometric decomposition (see Table 2) also evidences similar structural properties in the low- and high-resolution simulations. The bulge-disc parameters of HD-5004L are almost identical to those of HD-5004A (differences always smaller than $\sim 6$ per cent). The only exception is the Sérsic parameter $n$, which implies that the stellar distribution in the innermost region is less concentrated in the low-resolution run. The $D / T$ ratios (see Table 4 ) obtained from both photometric fits and a 'kk-means' decomposition are also rather similar to those measured in HD-5004A.

Kinematic properties do not appear significantly affected by resolution effects either. Indeed, the specific angular momenta for the low-resolution DM halo, stellar bulge and baryonic disc (red symbols in left-hand panel of Fig. 5) lie very close to their highresolution counterparts. Indeed, the specific angular momenta for the low-resolution baryonic disc (red symbols in right-hand panel of Fig. 5) lie very close to their high-resolution counterparts. The circular velocity curves of HD-5004L and HD-5004A are also quite similar, especially at large radii. As in other works (e.g. Scannapieco et al. 2011b), the major differences are located in the innermost regions, up to $6 \mathrm{kpc}$, and they are mainly due to the different distribution of mass. In particular, the peak circular velocity diminishes about 8 per cent in run HD-5004L. In this object the contribution of gas to the total curve is negligible, so the difference between maximum values is mainly due to the stellar distribution. Finally, in Fig. 4 we have also included the Tully-Fisher relation for the low-resolution counterpart of object HD-5004A, blue square dot. This object has a similar total magnitude but its baryons are rotating slower, so the final low-resolution galaxy is brighter for spirals of similar rotation speed.

In view of these results, we conclude that the low- and highresolution objects have converged in their structural and kinematic properties for the purpose of our analysis.

\section{THE FINE STRUCTURE}

\subsection{Dynamical decomposition method}

In order to characterize the properties of different components in the simulated galaxies, we have devised a procedure to segregate star particles into the main components, following the models proposed by Abadi et al. (2003b) and Scannapieco et al. (2009).

The existence of a thin gaseous disc defines a symmetry axis, the $z$-axis, determined by the angular momentum of gas particles in the inner regions of haloes at $z=0$. We use this axis to orientate the object and then compute the eccentricity parameter, $\epsilon_{J}$, as the ratio of the $z$-component of the angular momentum of each baryonic particle, $j_{z}$, and the angular momentum expected for a particle with the same binding energy in a circular orbit, $J_{\mathrm{C}}(E)$ (Abadi et al. 2003b). In Fig. 6 we plot the distribution of this parameter, $\epsilon_{J}$, for stars (black line) and gas (magenta line) in all simulated objects. As we would expect most gas particles are distributed in circular orbits, with $\epsilon_{\text {Jgas }} \sim 1$. The distribution of stars, on the contrary, typically shows two peaks: one at $\epsilon_{J *} \sim 1$ and a second one at $\epsilon_{J *} \sim 0$. This distribution is quite similar to that previously found by other authors (e.g. Abadi et al. 2003b; Scannapieco et al. 2009), and reveals the presence of two main components: a cold disc in rotational support and a hot spheroid supported by dispersion.

Although the distribution of $\epsilon_{J}$ allows us to infer the relative importance of disc and spheroid, it is not enough for classifying individual stars into different components. In order to assign individual stellar particles to the different galactic components (thin and thick discs, bulge and halo), more parameters are needed in addition to the eccentricity $\epsilon_{J}$. In this work we will also use the total binding energy, $E$, computed using all particles inside the virial radius, and the ratio of the projected angular momentum of each baryonic particle on the plane of the disc, and the angular momentum expected if the same particle was in a circular orbit $j_{p} / J_{C}$.

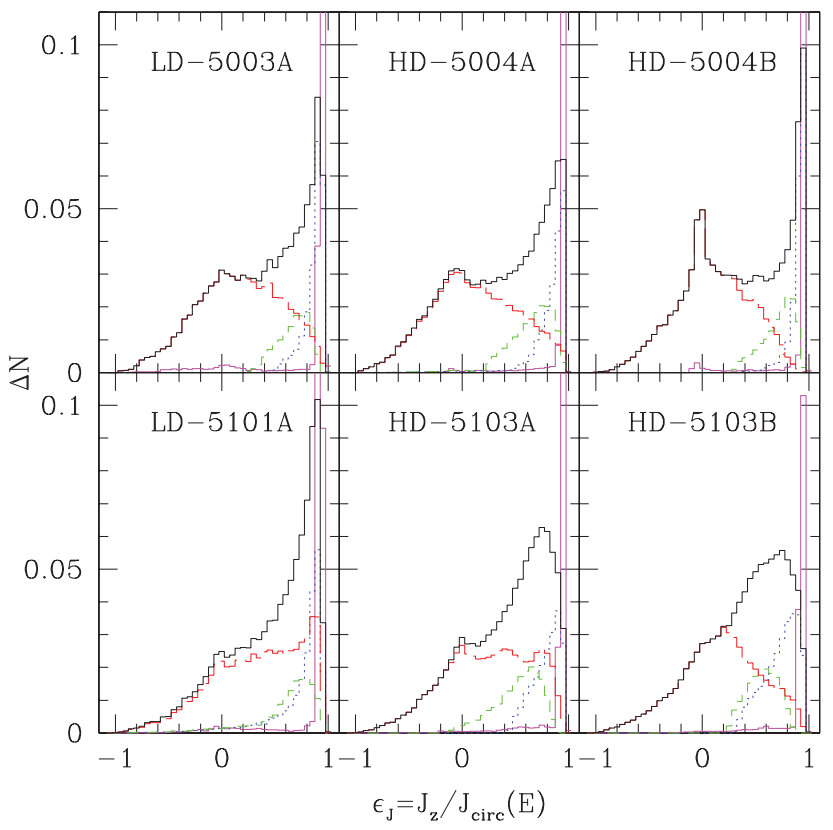

Figure 6. Mass fraction of stars (black) and gas (magenta) for simulated galaxies at $z=0$, as a function of $\epsilon_{J}=j_{z} / J_{\mathrm{C}}$. Blue dotted, green short dashed and red long dashed lines stand for mass fractions of stars in the thin disc, thick disc and spheroid as decomposed by 'kk-means' (see text for details). 


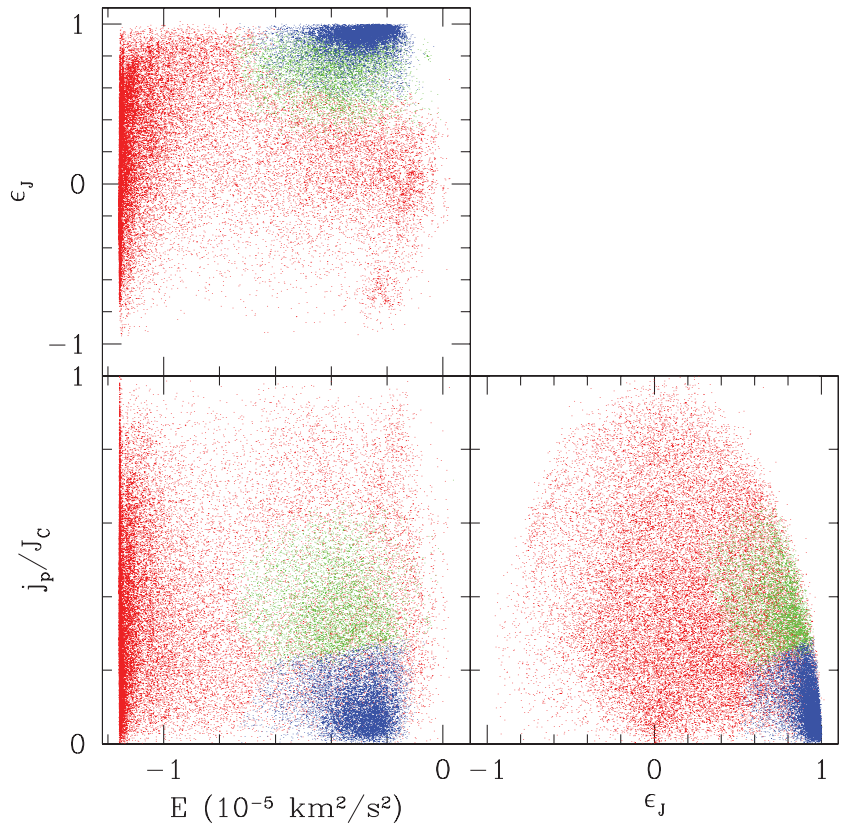

Figure 7. Thick disc stars (green), thin disc stars (blue) and spheroid stars (red) decomposed by 'kk-means' in the 3D space defined by $\epsilon_{J} j_{P} / J_{\mathrm{C}}$ and $E$, the total binding energy computed using all particles inside the virial radius, for simulated galaxy LD-5003A.

As it can be seen in Fig. 7, where we plot all the stars in object LD$5003 \mathrm{~A}$, there exist heavily populated regions in the space defined by these three parameters. Those regions can be easily identified as the spheroid and the thin disc. In addition we also can recognize an intermediate, well-populated region, which is also separated by means of these parameters and that we identify as the thick disc.

In order to assign stars in different components we use an unsupervised clustering algorithm, the 'kk-means' method (Schölkopf, Smola \& Müller 1998; Dhillon, Guan \& Kulis 2004; Karatzoglou, Smola \& Hornik 2004), which is the weighted kernel version of the 'k-means' algorithm. The 'kk-means' method uses the 'kernel trick' in order to implicitly project all data into a non-linear feature space using a Gaussian kernel. Since the points are now located in a vector space of greater dimensionality, it is able, in principle, to capture clusters that are not linearly separable in input space, but are so in feature space. The algorithm attempts to minimize the sum of the distance in feature space from points to the assigned cluster centres, in the same way the standard k-means algorithm does. It proceeds iteratively from an initial set ${ }^{4}$ of cluster centres by assigning each point to the cluster with the closest centre. Then, the centres are re-computed as the centroids of each cluster.

Given that the algorithm scales relatively poorly with the number of points and cluster centres, we do not apply the algorithm to all stars in the main object. Instead we select a random sample of points to classify according to this method. The rest of stars are classified computing the Euclidean distance in the feature space, and selecting the nearest neighbours decided by majority vote. Finally we decide, studying the region spanned by each cluster, which group corresponds to the different components of the object. We have

\footnotetext{
${ }^{4}$ We run several instances of the algorithm to ensure it properly converges to a stable set of points that is independent of the initial choice of cluster centres.
}

performed tests with different sample sizes to ensure the sampling procedure does not affect the clustering results.

The unsupervised nature of this segregation procedure implies that no prior, besides the expected number of components, has been assumed when separating the different components. In its construction we have also avoided the use of the properties (e.g. age or metallicity) expected for the stellar population of each component. Therefore, this decomposition method will allow for an unbiased study of many component properties and the connection with its history of mergers and accretion events in the cosmological context.

In Fig. 8 we show the edge-on synthetic images of all stars in each galaxy, including satellites that will be excluded for the subsequent analysis, in top panels, and in subsequent rows we show the edge-on projection of stars in different components: thin disc, thick disc, and spheroid, respectively. The $\epsilon_{J}$ distributions of stars in each of these identified components are shown in Fig. 6, using the same colour code as in Fig. 7. In this figure, we can see that the spheroid component (in red) shows almost no net rotation for most objects. Its distribution, however, tends to be skewed towards positive values of $\epsilon_{J}$, this being especially clear for object HD-5101A. We interpret this skewness as a manifestation of a possible dual origin of the bulge, with a classical component supported by velocity dispersion, and hence a symmetric $\epsilon_{J}$ distribution, and a pseudobulge component with a net rotation (Obreja et al. submitted). In object HD-5103, A and B, it is clearly seen that stars and gas peak at different $\epsilon_{J_{*}}$ values. This may indicate that the stellar disc is not aligned with the gas disc, probably due to the recent merger event in which it was involved.

Table 3 summarizes the main properties of each component. We see from this table that the fraction of stars assigned to the thick disc is $\sim 16$ per cent of the total stellar mass, in agreement with observational results (e.g. Yoachim \& Dalcanton 2006; Collins et al. 2011), in which the fraction of stars locked in thick disc ranges from $\sim 10$ to $\sim 35$ per cent. Columns (6) to (9) in this table show the scale lengths of the thin $\left(r_{s 1}\right)$ and the thick $\left(r_{s 2}\right)$ discs obtained by fitting their $r$-band luminosity profile with a simple exponential, and the goodness of those fits $\left(\chi^{2}\right)$. From these quantities we see that both disc components have similar radial scale lengths, as in observed galaxies (Yoachim \& Dalcanton 2006). Columns (10) to (13) show the scale heights $z_{\mathrm{h} 1}$ and $z_{\mathrm{h} 2}$ computed for thin and thick discs by just considering the fraction of red giant branch (RGB) stars in a solar cylinder, as it will be explained in Section 5.1. We find that for LD objects the mean values of thin and thick disc scale heights are lower than for objects in HD regions, as we would expect. In any case, the ratios of the scale heights for the thick and thin discs in our simulated galaxies are found to be about 4 (excluding HD-5103), with a large spread, as usually found for external galaxies (e.g. Yoachim \& Dalcanton 2006), and in our Galaxy (e.g. Gilmore \& Reid 1983; Siegel et al. 2002; Jurić et al. 2008). To end up, in the last column of Table 3 we display the asymmetric drift $\left(V_{\text {lag }}\right)$, computed as the difference between mean rotational velocity of thick and thin disc stars. These values show that the thick disc rotates at a slower pace than the thin disc as it occurs in our Galaxy (Soubiran et al. 2003) and in some external galaxies (Yoachim \& Dalcanton 2005).

Finally, in Table 4 we show different $D / T$ ratios measured with different techniques. $D / T^{\mathrm{P}}$ represents values obtained from $1 \mathrm{D}$ fits of the total $i$-band luminosity profile. The next column shows the $D / T_{\mathrm{kk}}^{\mathrm{P}}$ ratios obtained when the luminosity profile of particles in the 'kk-means' spheroidal component is fitted with a Sérsic function and, separately, the luminosity profile of particles in the 'kk-means' disc components is fitted with a single exponential. Finally, $D / T_{\mathrm{kk}}^{\mathrm{L}}$ 
LD-5003A

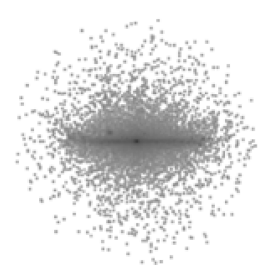

LD-5101A
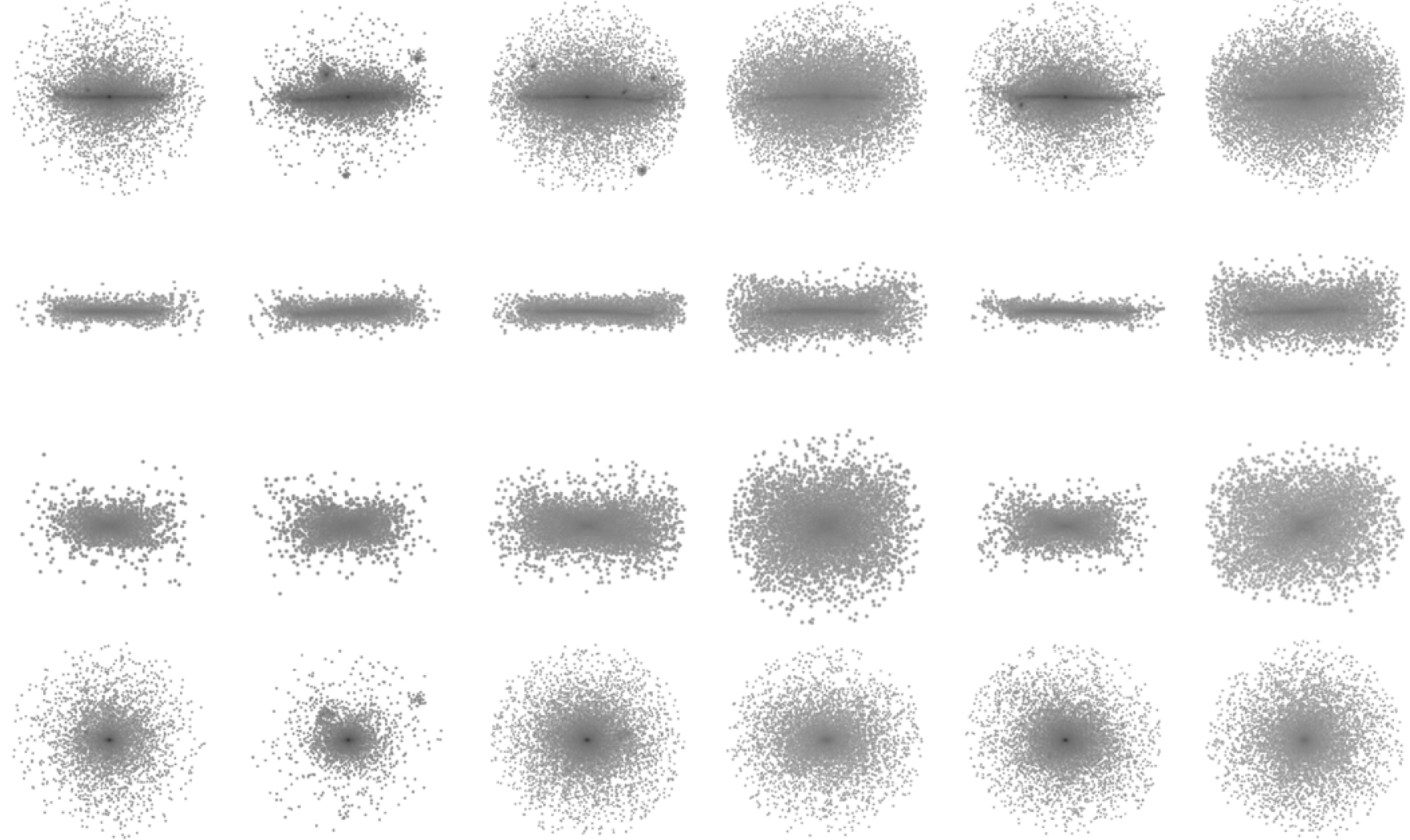

HD-5004B

HD-5103B
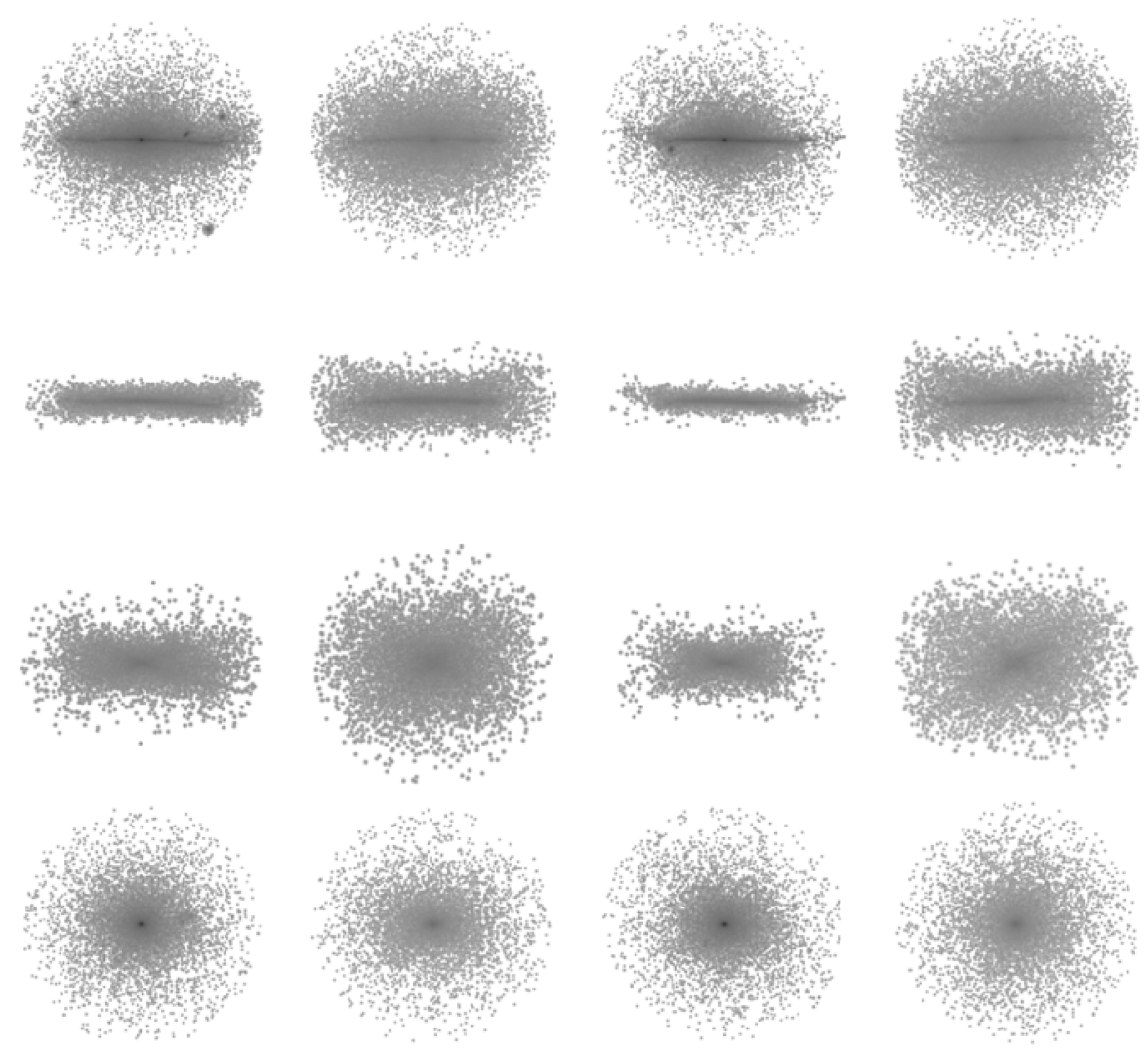

Figure 8. Edge-on maps of stellar magnitudes ( $r$ band) for the simulated galaxies at $z=0$. All images are $40 \mathrm{kpc}$ side. Top panels show the total stellar content of each object, including satellites that will be excluded for the analysis, and in next rows we draw, from second row to bottom, thin stellar disc, thick stellar disc and spheroidal component as classified by our method.

Table 3. Main properties of components: stellar mass enclosed in the spheroid $\left(M_{\text {sph }}\right)$, thin disc $\left(M_{\text {thin }}\right)$ and thick disc $\left(M_{\text {thick }}\right)$. Fraction of mass in the thick disc compared to the total stellar mass in the object $\left(f_{\text {thick }}\right)$. Scale lengths of the thin $\left(r_{s 1}\right)$ and thick $\left(r_{s 2}\right)$ disc components, and the goodness of the fits $\left(\chi^{2}\right)$. Scale heights of the thin $\left(z_{\mathrm{h} 1}\right)$ and thick $\left(z_{\mathrm{h} 2}\right)$ discs, and the goodness of the fits $\left(\chi^{2}\right)$. Finally, the asymmetric drift $\left(V_{\mathrm{lag}}\right)$.

\begin{tabular}{|c|c|c|c|c|c|c|c|c|c|c|c|c|c|}
\hline $\begin{array}{l}\text { Galaxy } \\
\text { (1) }\end{array}$ & $\begin{array}{c}M_{\mathrm{sph}} \\
10^{10} \mathrm{M}_{\odot} \\
(2)\end{array}$ & $\begin{array}{c}M_{\text {thin }} \\
10^{9} \mathrm{M}_{\odot} \\
\text { (3) }\end{array}$ & $\begin{array}{c}M_{\text {thick }} \\
10^{9} \mathrm{M}_{\odot} \\
\text { (4) }\end{array}$ & $\begin{array}{c}f_{\text {thick }} \\
\text { (5) }\end{array}$ & $\begin{array}{c}r_{s 1} \\
\mathrm{kpc} \\
(6)\end{array}$ & $\begin{array}{l}\chi^{2} \\
(7)\end{array}$ & $\begin{array}{c}r_{s 2} \\
\mathrm{kpc} \\
(8)\end{array}$ & $\begin{array}{l}\chi^{2} \\
(9)\end{array}$ & $\begin{array}{l}z_{\mathrm{h} 1} \\
\mathrm{kpc} \\
(10)\end{array}$ & $\begin{array}{l}\chi^{2} \\
(11)\end{array}$ & $\begin{array}{l}z_{\mathrm{h} 2} \\
\mathrm{kpc} \\
(12)\end{array}$ & $\begin{array}{l}\chi^{2} \\
(13)\end{array}$ & $\begin{array}{c}V_{\text {lag }} \\
\mathrm{km} \mathrm{s}^{-1} \\
(14)\end{array}$ \\
\hline D-5003A & 1.00 & 3.42 & 1.95 & 0.13 & 2.95 & 0.0022 & 2.87 & 0.0001 & 0.32 & 0.0291 & 1.49 & 0.0106 & -12.31 \\
\hline D-5101A & 0.52 & 3.1 & 1.69 & 0. & 3.83 & 0.0032 & 3.45 & 0.0002 & 0.45 & 0.0017 & 1.35 & 0.0102 & -4.87 \\
\hline HD-5004A & 1. & 5. & 5. & 0 & 4. & 0.0 & 3.81 & 0.0 & 0.42 & 0.0 & 2.33 & 15 & -16.53 \\
\hline HD- & 1.73 & 5.6 & 5.42 & 0.1 & 4.19 & & 4.26 & & 0.67 & & 8.32 & 0.0004 & -27.75 \\
\hline HD-5004B & 1.74 & 5.79 & 3.89 & 0.14 & 3.58 & 0.0027 & 3.39 & 0.0014 & 0.34 & 0.0059 & 2.17 & 0.0050 & -5.41 \\
\hline HD-5103B & 1.65 & 7.80 & 4.65 & 0.16 & 3.81 & 0.0002 & 4.76 & 0.0004 & 1.01 & 0.0005 & 13.4 & 0.0005 & -28.51 \\
\hline
\end{tabular}

Table 4. Disc-to-total ratios measured from different methods. See the text for an explanation of the different methods used for the computation of these $D / T$ values.

\begin{tabular}{lccc}
\hline Galaxy & $D / T^{\mathrm{P}}$ & $D / T_{\mathrm{kk}}^{\mathrm{P}}$ & $D / T_{\mathrm{kk}}^{\mathrm{L}}$ \\
\hline LD-5003A & 0.70 & 0.61 & 0.34 \\
LD-5101A & 0.83 & 0.75 & 0.44 \\
HD-5004A & 0.68 & 0.62 & 0.39 \\
HD-5103A & 0.68 & 0.56 & 0.40 \\
HD-5004B & 0.76 & 0.62 & 0.36 \\
HD-5103B & 0.56 & 0.57 & 0.49 \\
HD-5004L & 0.72 & 0.53 & 0.42 \\
\hline
\end{tabular}

gives the direct ratios, without any fit, of the 'kk-means' disc-to-total luminosity. We see from this table that the two fitted ratios $\left(D / T^{\mathrm{P}}\right.$ and $\left.D / T_{\mathrm{kk}}^{\mathrm{P}}\right)$ have roughly similar values for each object, with a systematic tendency for $D / T_{\mathrm{kk}}^{\mathrm{P}}$ to be slightly lower than $D / T^{\mathrm{P}}$. In contrast, the non-fitted direct ratios, $D / T_{\mathrm{kk}}^{\mathrm{L}}$, of the 'kk-means' components are significantly lower than $D / T^{\mathrm{P}}$. These results are in agreement with those found in Scannapieco et al. (2010), where our fitted and non-fitted $D / T$ ratios must be compared to their photometric and kinematic $D / T$ ratios, respectively. As noted by those authors, the difference obtained from these two methods can be partly explained from the fact that, in the 'kk-means' (or kinematic) decomposition, disc stars are not present or hardly present within the inner regions, while the fitted photometric decomposition assumes an 
exponential profile starting at the very centre of the galaxy. These results show that, although the photometric $D / T$ ratios can be employed to compare the properties of simulated and real galaxies, kinematic properties of galaxies are not properly inferred from such $D / T$ ratios (Scannapieco et al. 2010).

\subsection{Observational-like samples}

A full decomposition method like that described in the preceding Section 4.1, where each stellar particle is assigned to a galactic component, is very useful for the modelling of the galactic structure and formation processes, as well as for comparison with other simulation-based studies. However, it cannot be applied to observational data. In order to facilitate the comparison of our results with those available from observations, we have also selected spheroidal and disc subsamples based on two different criteria similar to those frequently used in observational studies. Each of these observational-like samples is not intended to contain all the stellar particles of a given component, but just a subset of particles easily identified in observations and that mostly belong to such a galactic component.

The first criterion is mainly founded on the positions of star particles in the galactic object. The bulge sample consists of particles in the innermost region $\left(r \leq 5 r_{\mathrm{e}}\right)$ above and below the galactic plane $\left(2 z_{\mathrm{h} 1} \leq|z| \leq 5 z_{\mathrm{h} 1}\right)$ to minimize the contribution of disc stars. The two disc samples are selected in a cylindrical shell $2 r_{\mathrm{s}} \leq r \leq$ $3 r_{\mathrm{s}}$, which minimizes the contribution from bulges, imposing a minimum rotation speed of $50 \mathrm{~km} \mathrm{~s}^{-1}$ in order to minimize the contribution from halo stars (Sales et al. 2009). The distance to the galactic plane determines the difference of both samples: stars located close to this plane $\left(|z| \leq 2 z_{\mathrm{h} 1}\right)$ constitute the thin disc sample, while those at farther heights $\left(z_{\mathrm{h} 2} \leq|z| \leq 3 z_{\mathrm{h} 2}\right)$ constitute the thick disc sample.

The second method uses kinematic information to select disc stellar samples, as employed in Galactic stellar studies (Bensby et al. 2003; Soubiran et al. 2003). In those works, thin and thick disc stars are selected by assuming that the Galactic space velocities of such stellar populations have Gaussian distributions

$f(U, V, W) \propto \exp \left(-\frac{U^{2}}{2 \sigma_{U}^{2}}-\frac{(V-\langle V\rangle)^{2}}{2 \sigma_{V}^{2}}-\frac{W^{2}}{2 \sigma_{W}^{2}}\right)$,

where $U, V$ and $W$ are the radial, azimuthal and axial components of the stellar velocity, respectively, and $\sigma_{U}, \sigma_{V}$ and $\sigma_{W}$ the velocity dispersions found for thin and thick disc Galactic stars (see e.g. Soubiran et al. 2003). Knowing these values, stars in the Galactic disc are classified according to their major probability of belonging either to the thin or to the thick disc. In our simulations, however, we cannot use the values of the Milky Way velocity dispersions, being necessary to compute them. To do that, we first selected a sample of stars in 'solar neighbourhood volumes', equivalent to cylindrical shells normal to the disc plane between two and three scale lengths of the thin disc $\left(2 r_{\mathrm{s}} \leq r \leq 3 r_{\mathrm{s}}\right)$, which will be mainly populated by disc stars, and then we segregated them in two components according to their kinematics and metallicity, as in Soubiran et al. (2003). These two new samples are employed to compute the characteristic velocity dispersions that we will apply in the classification of stars in the disc, not only in the 'solar neighbourhood volume'. According to Bensby et al. (2003), in order to minimize the contribution of the thin disc stars in the thick sample (and vice versa), we classify as thick disc stars those which are at least 10 times more likely to be a thick disc star than a thin disc star, i.e. those with $f_{\mathrm{TD}} / f_{D} \geq 10$. Similarly, thin disc stars are selected as those with $f_{\mathrm{TD}} / f_{D} \leq 0.01$.

\section{VERTICAL DENSITY PROFILES}

\subsection{Scale heights of the disc components}

The variation of stellar density with the distance $z$, above or below the Galactic plane, can be determined by counting faint stars in fields centred on the Galactic poles. Stellar counts in the solar neighbourhood result in vertical profiles which can be fitted with a double exponential function. This fact was interpreted by Gilmore \& Reid (1983) as due to the presence of two different disc components, thin and thick, embedded in the Galaxy. Values measured for this double fit rise to $\sim 350 \mathrm{pc}$ for thin disc scale height, and range from 750 to $1450 \mathrm{pc}$ for the thick one (e.g. Gilmore \& Reid 1983; Siegel et al. 2002; Jurić et al. 2008). In external galaxies, however, resolving stars is only possible for a small number of galaxies, but the presence of thick discs has been photometrically detected as an excess flux at large galactic latitudes (Dalcanton \& Bernstein 2002; Yoachim \& Dalcanton 2006). These works show that thick discs are ubiquitous in disc galaxies with scale heights spanning a wide range of values, but larger than the scale height of the embedded thin discs by a factor $\sim 2$. Ibata et al. (2009) measured the scale height for the Milky Way analogue NGC 891 from stellar counts and obtained a value, $z_{\mathrm{h}}=1.44 \pm 0.33 \mathrm{kpc}$, quite similar to that observed in the Galaxy.

In order to compute the vertical density profiles of simulated galaxies, we have followed a procedure similar to that commonly used in observational studies. The contribution of spheroidal components was minimized by just considering stellar particles with projected galactocentric distances in the range $2<r / r_{\mathrm{s}}<3$. We then counted the number of RGB stars implied, for the assumed IMF, by the age and metallicity of the stellar particles lying within different vertical bins. The vertical profile of the surface density was then obtained for each simulated galaxy by averaging several edge-on projections. Such profiles are shown (black solid lines) in the left-hand panels of Fig. 9, where we also represent the contribution of the thin (blue squares) and thick (green triangles) discs and the spheroid (red pentagons) decomposed by 'kk-means'. The corresponding exponential fits of both disc components are also shown (red lines) in Fig. 9, and their values are summarized in Columns (10) and (12) of Table 3. Fits of the thin disc take into account only the innermost part of the profile, similarly the thick disc fits ignore the innermost part, dominated by the thin disc.

The scale heights obtained in our LD objects have mean values of $\sim 0.4 \mathrm{kpc}^{5}$ for the thin disc and $\sim 1.4 \mathrm{kpc}$ for the thick component. In the HD objects these scales are somewhat higher, especially those of the thick discs, which have scale heights significantly larger than $2 \mathrm{kpc}$. This is likely due to the more active history of mergers and accretions for galaxies in crowded regions. Indeed, the object HD-5103A (and its counterpart HD-5103B with less SF efficiency) has suffered a recent merger and for this reason it is perturbed at $z=0$. As can be seen in the figure its vertical profile is almost exponential and it is dominated by thick disc stars, even at farther heights where halo stars are supposed to be the dominant population (red pentagons in Fig. 9). In any case, the ratios of the scale heights for the thick and thin discs $\left(z_{\mathrm{h} 2} / z_{\mathrm{h} 1}\right)$ in our simulated galaxies are found to be about 4, with a large spread, as usually found for external early spirals (see e.g. Yoachim \& Dalcanton 2006, focused on Sd galaxies, but containing a compilation for $\mathrm{S} 0-\mathrm{Sb}$ galaxies).

\footnotetext{
${ }^{5}$ Note that thin disc scale height is close to our gravitational resolution, and thus it should be regarded with caution.
} 
LD-5003A

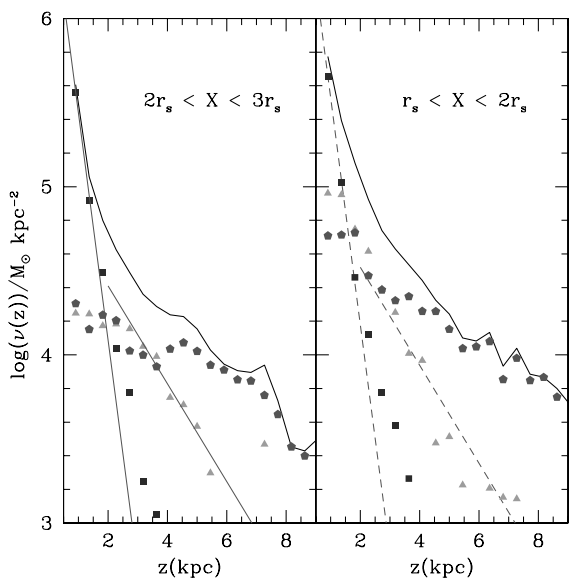

LD-5101A

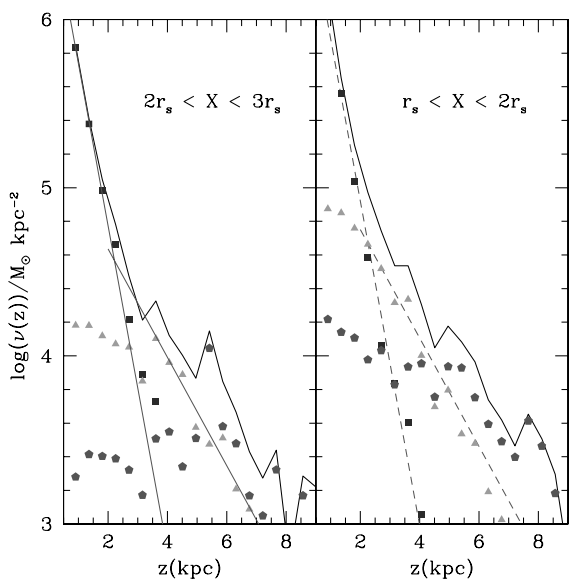

HD-5004A

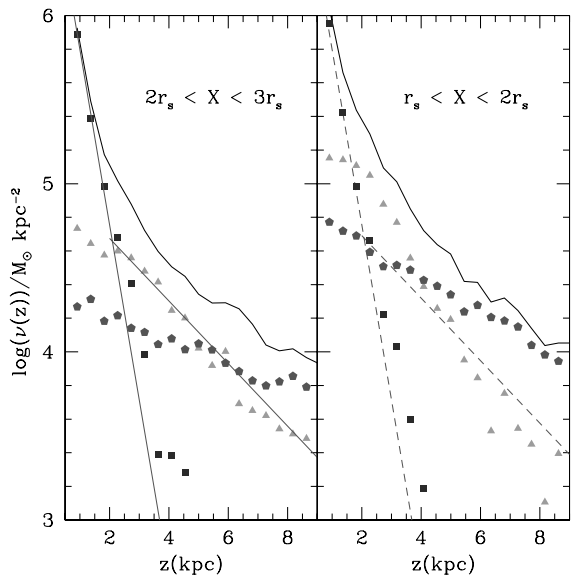

HD-5103A

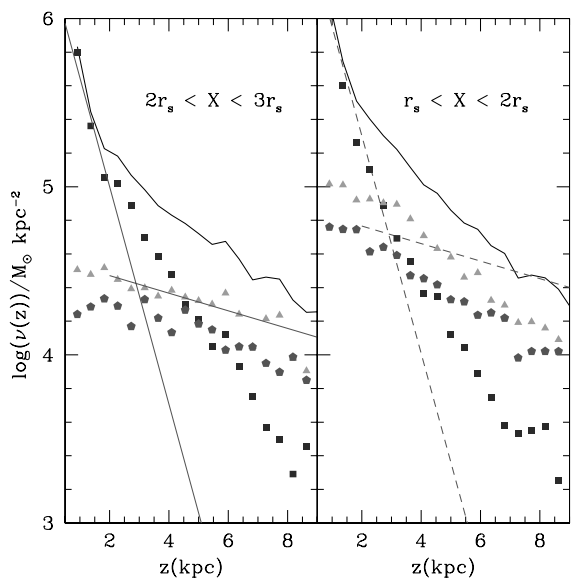

HD-5004B

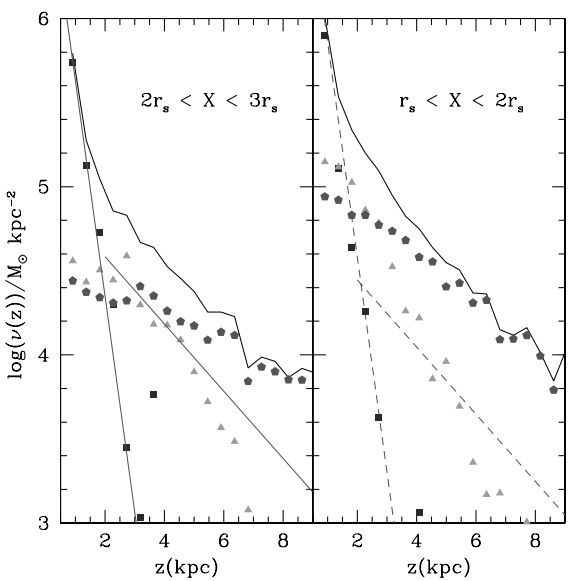

HD-5103B

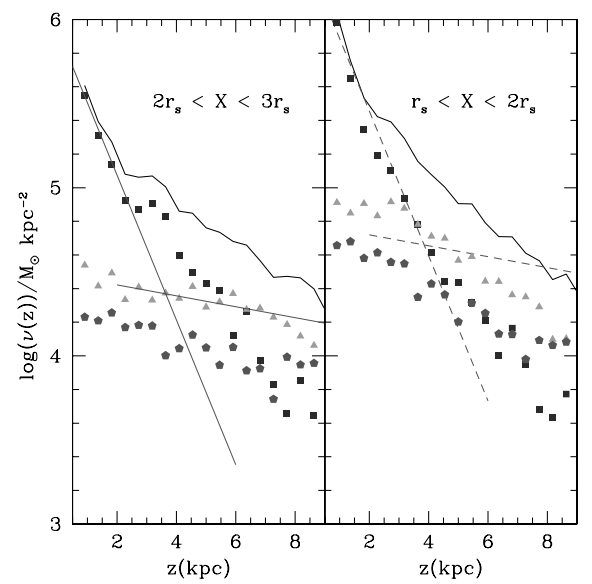

Figure 9. Vertical density profiles of all stars within two vertical cuts of the same width and different distance to the galactic centre. Black solid lines, blue squares, green triangles and red pentagons represent the total, thin disc, thick disc and spheroid stellar counts in each section, respectively. Red solid lines at the left-hand panels stand for the fits of thin and thick discs in the outermost region. Red dashed lines at the right-hand panels show an exponential fit to the profiles in the internal cut constrained to have the same scale heights for both the thin and thick discs, as those respectively measured in the outermost region.

\subsection{Radial variation of the disc scale heights}

Another important feature of observed thick discs is that their vertical profiles do not significantly depend on the galactocentric distance, i.e. the scale height seems to be roughly constant with radius (Robin et al. 2003; Ibata et al. 2009). Such a result is difficult to be reconciled with a merger-induced scenario for thick disc formation, which inevitably leads to flared thick discs (Bournaud et al. 2009), and favours models where the disc thickening results from internal processes such as gravitational instabilities.

In order to test whether the scale height changes with radius, we have followed the procedure employed by Ibata et al. (2009) for an observed external galaxy. We have drawn (right-hand panels of Fig. 9) the vertical density profile of stars within a region $(1<$ $\left.r / r_{\mathrm{s}}<2\right)$ inner than in the preceding analysis. For this internal region we have computed the exponential fits of both disc components constrained to have the same scale heights, $z_{\mathrm{h} 1}$ and $z_{\mathrm{h} 2}$, as in the external one. We find that, for the simulated objects (LD-5003A and LD-5101A) in LD regions of the universe, roughly constant $z_{\mathrm{h}}$ values would result in acceptable fittings for both the inner thin and thick. By contrast, for objects in HD regions (HD-5004A and HD-5103A, and their counterparts HD-5004B and HD-5103B with less $\mathrm{SF}$ efficiency), roughly constant $z_{\mathrm{h}}$ values give acceptable fits only for the thin disc of some simulated galaxies, but clearly fails for the thick disc. Therefore, for these latter objects we cannot reject the merger-induced scenario as a key ingredient for thick disc formation.

\section{STELLAR AGES}

\subsection{Age distributions of the different components}

Fig. 10 shows the distribution of stellar ages for the different components of our simulated galaxies. Each distribution was normalized to the total stellar mass of the corresponding individual component. The different rows in this figure depict the age distribution of samples selected from the different criteria described in Section 4: the first row refers to 'kk-means' decompositions, the second row to observational-like samples built from position criteria and the third row to observational-like samples built from kinematic criteria. The corresponding median values of stellar ages are summarized in Table 5, while the SF histories for each object and its 'kk-means' components are shown in Fig. 11. Note that, for runs LD-5101A and HD-5103B, the observational-like samples for the bulge component were too small for statistics and, hence, they are not depicted in Fig. 10. 


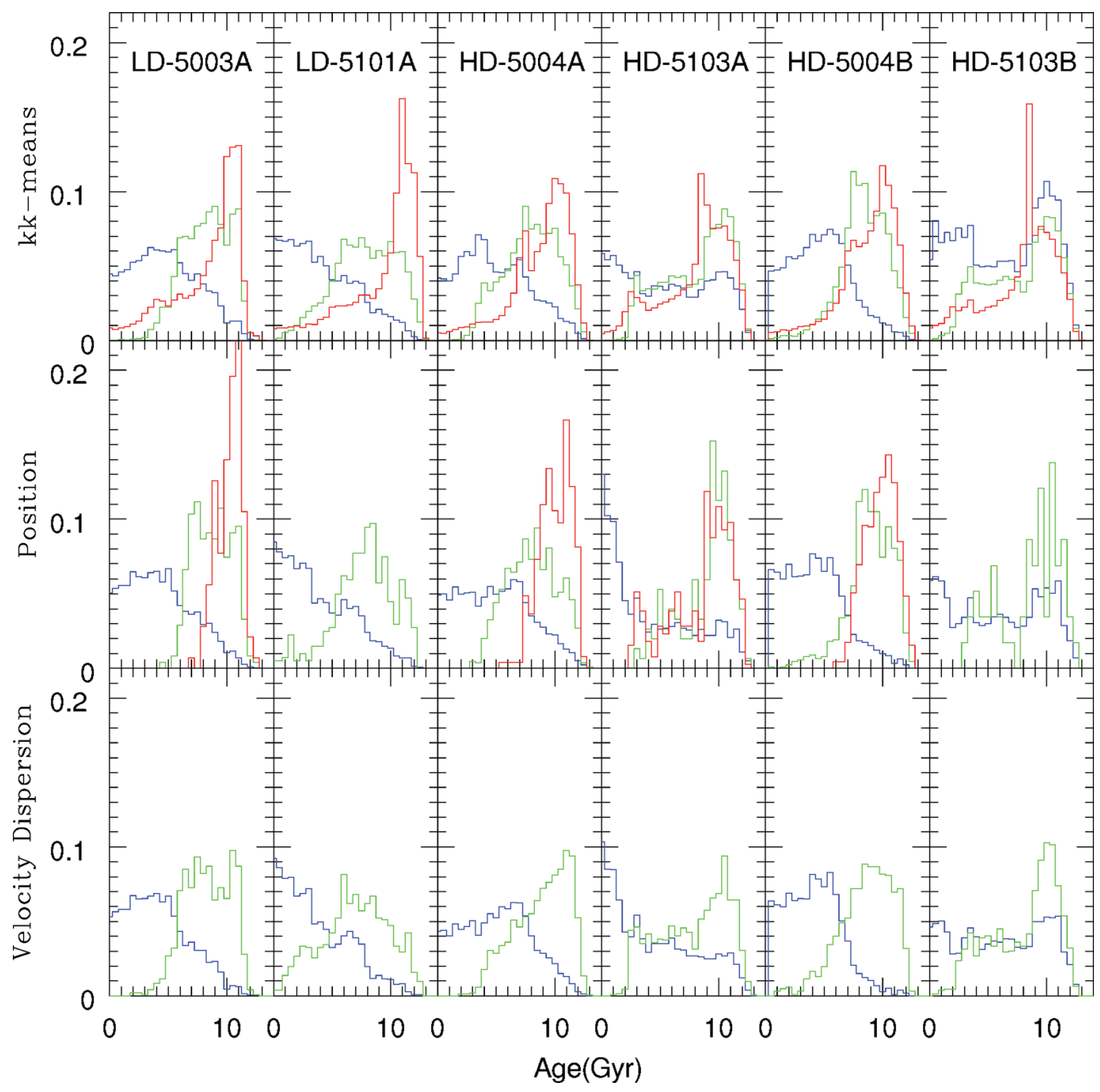

Figure 10. Stellar age distribution normalized to the total mass of each individual component. In each row we depict the age distributions obtained with different classification methods, as indicated in the $y$-axis. In the first row we show age distributions for star particles in each component identified by our classification method. In the second one samples are selected by positions in the simulated galaxies, and in the last one, kinematic criteria have been employed to identify stars in thin and thick discs. In all panels, the blue line refers to thin disc star particles, green is for thick disc and the red line stands for the spheroid.

A clear trend is found in all simulated galaxies for any kind of sample. The thin disc is always the youngest component with median ages ranging from 3.8 to $6.7 \mathrm{Gyr}$. Just a small fraction of the thin disc stars have ages older than $10 \mathrm{Gyr}$, while just a small fraction of stars in the spheroid are younger than 5 Gyr. The thick disc is instead populated by stars with intermediate ages. These results are consistent with the observational estimates for the different components in the Milky Way and nearby galaxies. Indeed, several (e.g. Fuhrmann 1998; Bensby et al. 2003, 2005; Reddy et al. 2006) agree that stars in the thick disc of the Galaxy have mean ages greater than 8 Gyr meanwhile the estimates for the thin disc stars lie between 5 and 7 Gyr. Bensby et al. (2005) derived a mean age of $4.3 \pm$ 2.6 Gyr for stars in the thin disc of the Galaxy, and 9.7 $\pm 3.1 \mathrm{Gyr}$ for a sample of thick disc stars. Similarly, for nearby galaxies, photometric studies find that the off-plane regions are dominated by old stars (>6 Gyr) (Dalcanton \& Bernstein 2002; Yoachim \& Dalcanton 2006). In their study of the integrated spectra of six galaxies, Yoachim \& Dalcanton (2008) obtained a median age of 4.6 Gyr for the thin disc, and ages ranging from 3.8 to $10.9 \mathrm{Gyr}$ (with a median value of 7.2 Gyr) for the thick disc. Scannapieco et al. (2011b) have also found similar results for the study of stellar populations in their simulated galaxies. The agreement between results obtained with different simulation techniques and decomposition methods could be interpreted as a general feature in the age of stellar populations of simulated galaxies.

Focusing on SF histories, we can see in Fig. 11 that the thin discs of all simulated galaxies are currently forming stars with a SF rate lower than about one solar mass per year. For almost all simulations the bulk of stellar mass formed $10 \mathrm{Gyr}$ before the present epoch, populating preferably the spheroid. At low redshift, however, there is just some residual SF in this component. On the contrary, the thick disc is not forming new stars at low redshifts. The stellar particles settled in this component were formed during an extended epoch in the past, with a low rate similar to that observed in the thin disc. In the last $10 \mathrm{Gyr}$ merger and accretion events triggered the SF leaving a trace as peaks in the SF history. This is clearly visible in simulation HD-5103A and its counterpart HD-5103B, which have been involved in a recent major merger. The new stars 
Table 5. Stellar median ages in Gyr for different components of each simulated galaxy. The three rows for each object correspond to a 'kk-means' decomposition, samples from position criteria and samples from kinematic criteria, respectively.

\begin{tabular}{cccc}
\hline Galaxy & Age $_{\text {sph }}$ & Age $_{\text {thick }}$ & Age $_{\text {thin }}$ \\
\hline LD-5003A & $9.6 \pm 2.2$ & $8.7 \pm 2.3$ & $4.7 \pm 3.3$ \\
& $10.7 \pm 1.0$ & $9.0 \pm 2.0$ & $4.3 \pm 3.1$ \\
& & $8.5 \pm 2.3$ & $4.1 \pm 2.9$ \\
LD-5101A & $10.8 \pm 1.9$ & $8.1 \pm 3.0$ & $3.8 \pm 3.3$ \\
& & $8.1 \pm 2.4$ & $3.3 \pm 3.2$ \\
HD-5004A & $9.7 \pm 2.2$ & $8.3 \pm 2.5$ & $4.7 \pm 3.3$ \\
& $10.3 \pm 1.6$ & $8.3 \pm 2.4$ & $5.0 \pm 3.6$ \\
HD-5103A & $9.0 \pm 2.9$ & $9.0 \pm 2.8$ & $5.7 \pm 3.8$ \\
& $9.4 \pm 2.7$ & $9.8 \pm 2.3$ & $3.1 \pm 3.8$ \\
HD-5004B & $9.5 \pm 2.2$ & $8.6 \pm 1.9$ & $4.9 \pm 2.7$ \\
& $10.2 \pm 1.5$ & $9.3 \pm 1.8$ & $4.3 \pm 2.7$ \\
HD-5103B & $8.8 \pm 2.0$ & $8.7 \pm 2.9$ & $6.7 \pm 3.7$ \\
& $9.9 \pm 1.8$ & $9.4 \pm 2.7$ & $6.2 \pm 3.8$ \\
& & $9.2 \pm 2.8$ & $6.4 \pm 3.7$ \\
\hline
\end{tabular}

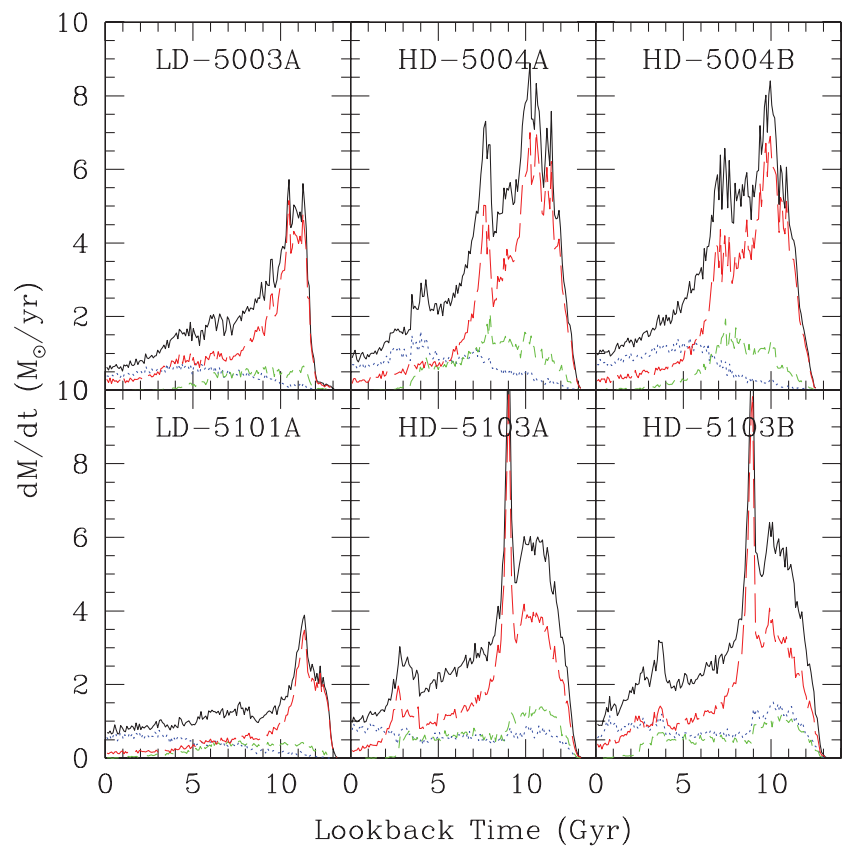

Figure 11. SF history for each component. Black solid line stands for the total SF rate in the object. Thin disc is represented with a blue dotted line, and green short dashed line for the thick disc. SF rate in the spheroidal component is plotted with a red long dashed line.

formed during this event mainly increase the stellar content in the spheroid and the thin disc. Differences in the SF parameters produce differences in the response of simulated galaxies to merger events. In those objects in which SF has been difficult, the SF occurs more quietly and during a more extended epoch than in their counterparts with a more efficient SF, in which those events produce sharper peaks.

\subsection{Age and colour gradients}

Colour profiles are related with galactic properties such as metallicity, stellar age or dust content, and for this reason they have long been used for exploring galactic properties in large galaxy samples and catalogues (e.g. Gonzalez-Perez, Castander \& Kauffmann 2011). Fig. 12 shows the $g-r$ colour and age profiles for each simulated galaxy. Black solid lines depict the total profiles, while coloured dashed lines show the individual profiles of each galactic component identified by the 'kk-means' method. From this figure we see that the colour profiles are dominated by the thin disc contribution, which is responsible for their U-shape: every galaxy and thin disc in our sample is redder in the innermost region and becomes bluer as we move away in the radial direction until a minimal value is reached. Then, the stellar content becomes progressively redder. These results are in good agreement with observations of Type-II spiral galaxies (e.g. Bakos, Trujillo \& Pohlen 2008; Yoachim \& Dalcanton 2008; Gonzalez-Perez et al. 2011), where such U-shaped profiles are also found, with median colour gradients close to zero. These features have been recently studied using cosmological hydrodynamic simulations (Martínez-Serrano et al. 2009; Sánchez-Blázquez et al. 2009) and tend to favour an inside-out formation scenario for the galactic discs. According to such a scenario, SF has moved progressively towards outer regions of galactic discs. As a consequence, older and more metal-rich stellar populations are located in the innermost regions of discs, leading to negative colour gradients. Due to the secular evolution, stars migrate from the inner to the outer parts of the galaxy, reaching the outskirts where the specific SF is very small. These outskirts progressively become populated by old and red stars (Martínez-Serrano et al. 2009; Sánchez-Blázquez et al. 2009).

In contrast to that found for the thin disc, the colour and age profiles depicted in Fig. 12 for the thick disc and spheroidal components are quite uniform across the galaxy, with little sign of radial gradient. These profiles are a clear indication of a different formation scenario for such components: their stellar content is older and was formed in a shorter time than in the thin disc (see also Fig. 10 of age distributions). As in observed galaxies (MacArthur, González \& Courteau 2009), age gradients in the spheroid are almost zero, with just small deviations from a flat profile towards both negative and positive values. These deviations are probably more visible for objects in LD regions, where the spheroid shows a slightly negative age gradient.

\section{CHEMICAL PROPERTIES}

\subsection{Metallicity distribution functions}

Element abundances of individual stars and gas provide a valuable record of the SF and enrichment history during the early stages of galaxy formation and evolution, and it has long been used to propose or test the different mechanisms involved in such processes (e.g. Gilmore et al. 1995; Reddy et al. 2006; Schuster et al. 2006). The fact that kinematic and chemical properties of thick disc stars differ form those of thin disc stars has been interpreted as evidence of their distinct nature, making possible the differentiation of stars from both components using their chemical properties (e.g. Schuster et al. 2006; Schönrich \& Binney 2009b). Otherwise Meléndez et al. (2008) found no significant abundance differences between the bulge and the local thick disc stars in their study, which is interpreted as a consequence of the rapid SF history of both components, rather than any deeper relation between them. 


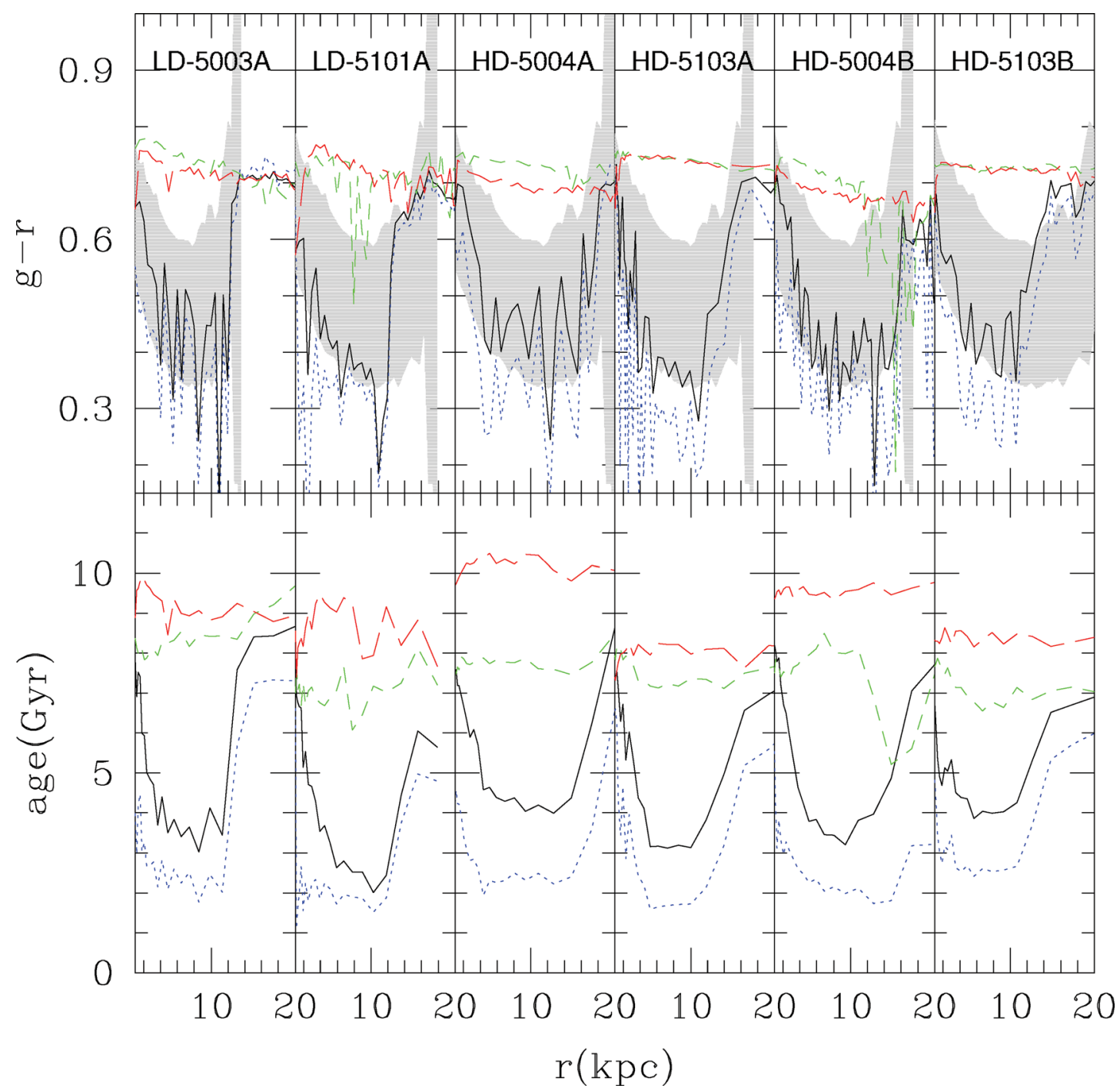

Figure 12. Top panels: colour profiles for each component in the galaxy as seen face-on. As in previous figures solid line stands for the colours of all stars in the galaxy. Thin disc stars are plotted using a blue dotted line, and the colour gradient of thick disc stars is shown with a green short dashed line. Red long dashed line stands for colour gradients in spheroid stars. For reference, the observed colour profiles of 39 Type-II galaxies, as reported by Bakos et al. (2008), are shown as a shaded grey area. Note that the population synthesis used in this paper does not include dust effects. Bottom panels: mean stellar ages weighted by $r$-band luminosity for each simulated galaxy. Line code is as in the top panel and previous figure. Similar profiles weighted by mass can be found in our previous paper (Martínez-Serrano et al. 2009).

In the Milky Way, thick disc stars have been found to be old and more metal-poor and also $\alpha$-enhanced compared to stars in thin disc (e.g. Fuhrmann 1998; Bensby et al. 2003, 2005; Reddy et al. 2006; Ruchti et al. 2010). To constrain the chemical evolution of the Galaxy, samples of long-lived stars, as F and G dwarfs, have been employed (e.g. Gilmore et al. 1995; Fuhrmann 1998; Bensby et al. 2003, 2005; Reddy et al. 2006). The metal distribution of thick disc stars is roughly characterized as a Gaussian with mean iron abundance $\sim-0.7$ to -0.5 dex (e.g. Gilmore et al. 1995; Schuster et al. 2006), with most stars between -0.5 and -1.0 dex, but with a long tail of metal-poor stars which extends to about $-2.2 \mathrm{dex}$, values usually associated with halo stars. This metal-poor tail is interpreted by some authors as evidence of the complex structure within the thick disc (e.g. Schuster et al. 2006; Carollo et al. 2010). Yoachim \& Dalcanton (2008) used Lick metallicity-sensitive indices to derive metallicity properties of thin and thick disc components in six external galaxies. They found metal-poor and no significant $\alpha$-enhanced thick discs, probably due to the insensitivity of Lick indices to the differences found at low metallicities. The median metallicity value measured in the thick and thin discs of these galaxies is $[\mathrm{Z} / \mathrm{H}]=$ -0.6 , as expected for low-mass galaxies.

In Fig. 13 we depict the metallicity distribution function (MDF) of each component in our simulated galaxies. ${ }^{6}$ As in Fig. 10 each row corresponds to samples selected from the different criteria described in Section 4, and each distribution is normalized to the total stellar mass of the corresponding individual component. In general we find that the thin disc is the most metal-rich component, with median values close to the solar value, between -0.19 and -0.06 . Moreover, the distribution is quite similar for the different thin disc samples. The spheroid, on the contrary, is the most metal-poor distribution, with median values covering a wide range between -0.61 and

\footnotetext{
${ }^{6}$ The solar abundances of Grevesse \& Sauval (1998), with $\mathrm{Z}_{\odot}=0.0169$, have been assumed for the subsequent analysis.
} 


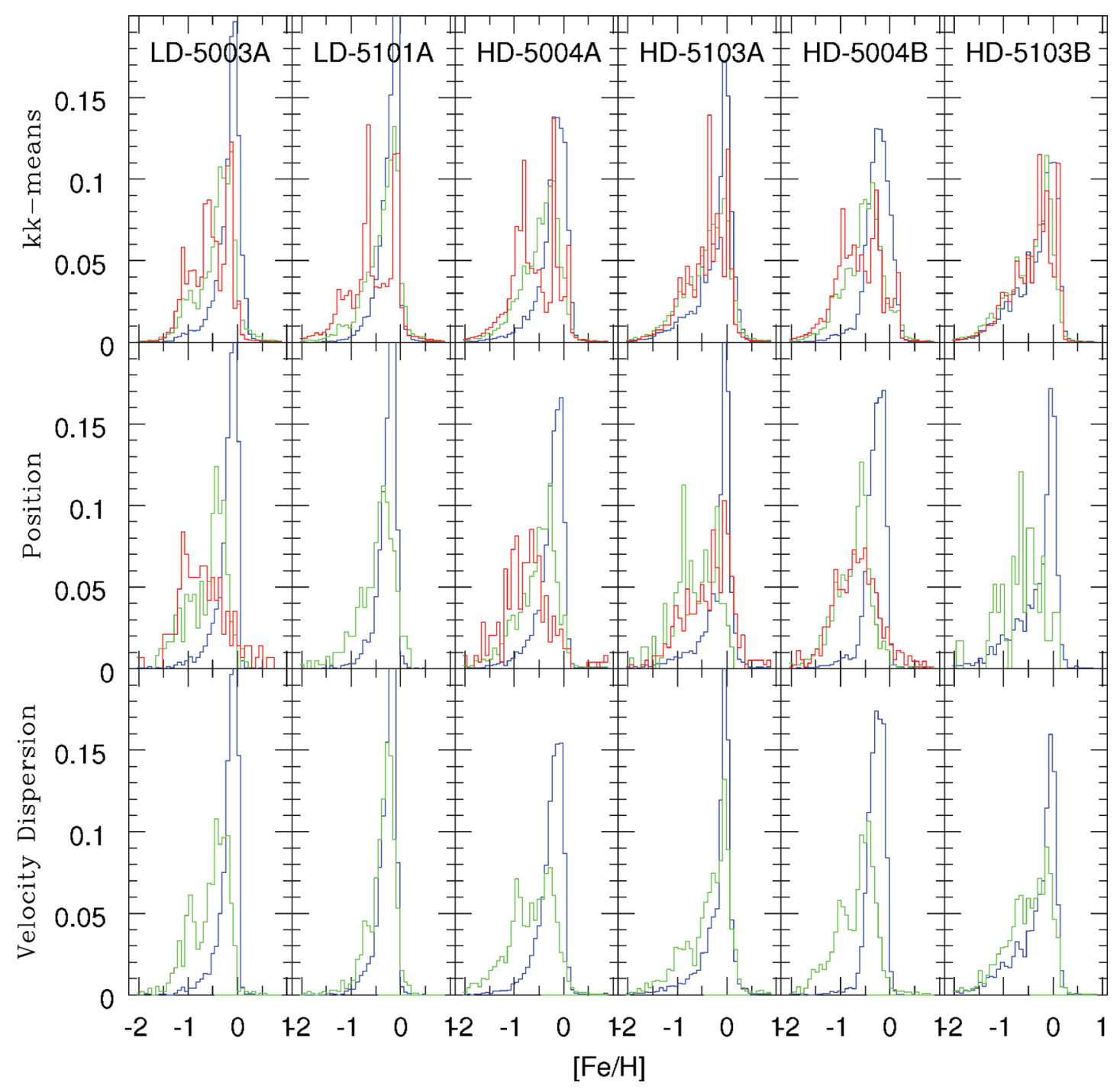

Figure 13. Metallicity distribution function of stars in each galaxy component. As in Fig. 10 in each row is depicted the MDF of samples selected from the different described criteria. Each component is represented by different colours, as in previous figures.

-0.27. In this component a clear difference is observed among the different samples. In those based on the 'kk-means' method we find a bimodal MDF, while such a bimodality is not observed in the samples selected from position criteria, which exclude stars near the galactic plane. Since the distribution of parameters $\epsilon_{J}$ and $j_{p}$ for these high-metallicity stars in the spheroid clearly corresponds to this component, such a high-metallicity peak seems to be a real feature of the spheroid MDF, and not an artefact due to disc stars that eventually could be misclassified as belonging to the spheroidal component. The bimodal MDF for the spheroid seems more likely due to the different formation mechanisms of the bulge of spiral galaxies: the low-metallicity peak would correspond to the classical bulge, while the high-metallicity peak would correspond to the bulge fraction formed from instabilities of the inner disc (Obreja et al. submitted).

With the only exception of simulation HD-5103A and its counterpart HD-5103B, in which the stellar content is well mixed, the thick disc MDF shows an intermediate behaviour between the thin disc and the spheroid. This component has in fact a metal content poorer than in the thin disc, while richer than in the spheroid. Their MDFs peak at values from -0.45 to -0.24 , but their shape is not as sharp as for the thin disc and extends to very metal poor regions. In the case of observational-like samples selected from kinematic criteria (third row in Fig. 13) the thick disc MDF shows a clear bimodality, which could be related with the metal-poor thick disc defined by some authors (Schuster et al. 2006; Carollo et al. 2010).

\subsection{Metallicity variation along the disc}

The distribution of element abundances within galaxies is another important feature in the comprehension of galaxy formation and evolution. First measures, based in emission lines in large samples of H II regions in spiral galaxies (e.g. Shaver et al. 1983; Zaritsky, Kennicutt \& Huchra 1994; Garnett et al. 1997; van Zee et al. 1998), found radial abundance gradients in almost all large spiral galaxies, with inner H II regions richer than outer ones. Shaver et al. (1983) measured an oxygen abundance gradient of $\sim-0.07 \mathrm{dex} \mathrm{kpc}{ }^{-1}$ in the Milky Way, a value quite similar to that obtained by Luck, Kovtyukh \& Andrievsky (2006) fitting iron abundances of young stars in the Galaxy, or Pancino et al. (2010) using open clusters. This result suggests that the chemical evolution of the Galactic disc is determined mainly by local chemical evolution, because mergers 

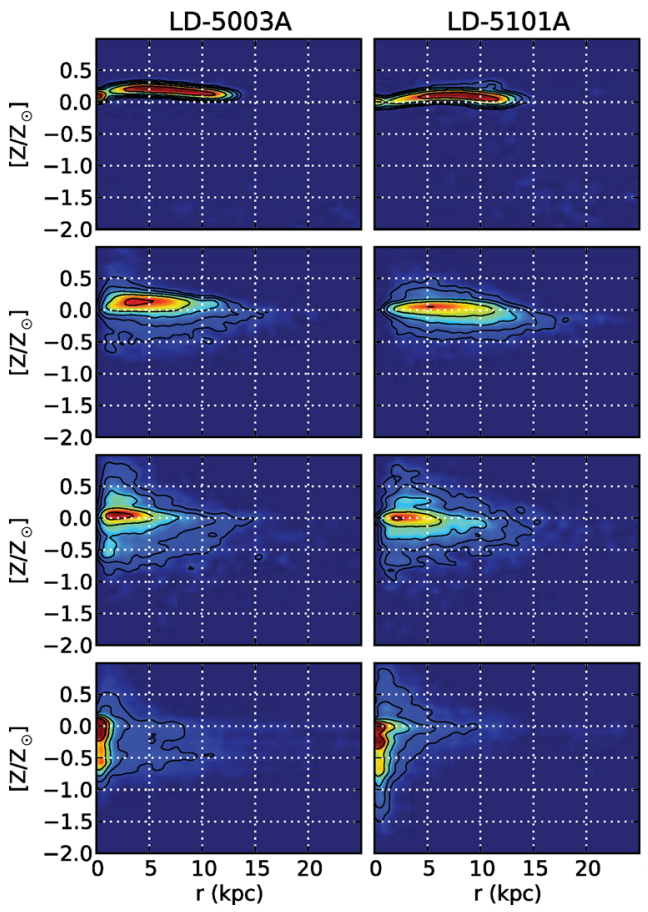
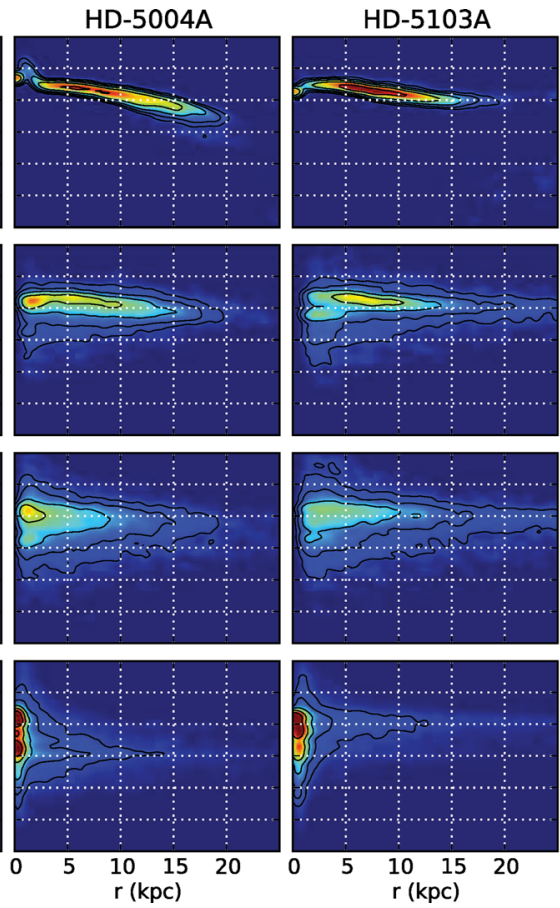

HD-5004A
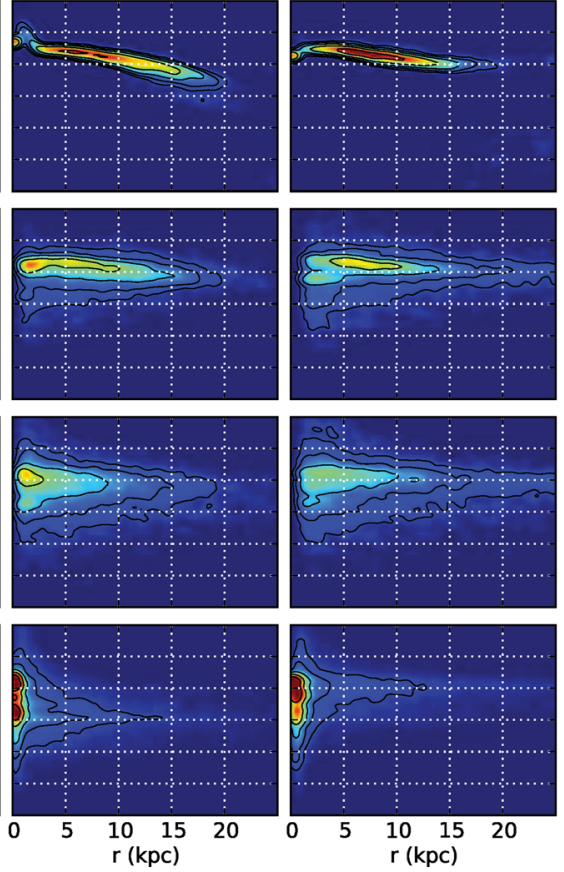

HD-5004B
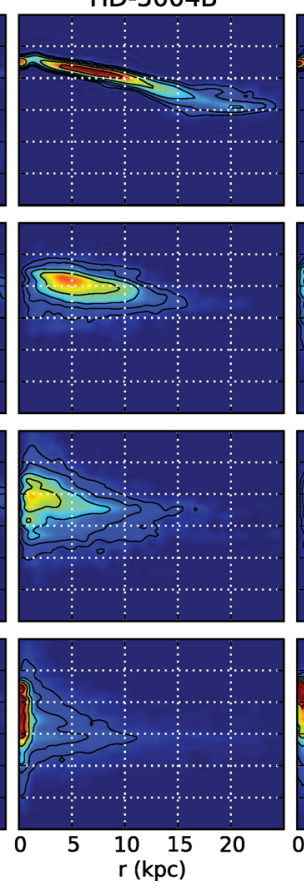

HD-5103B
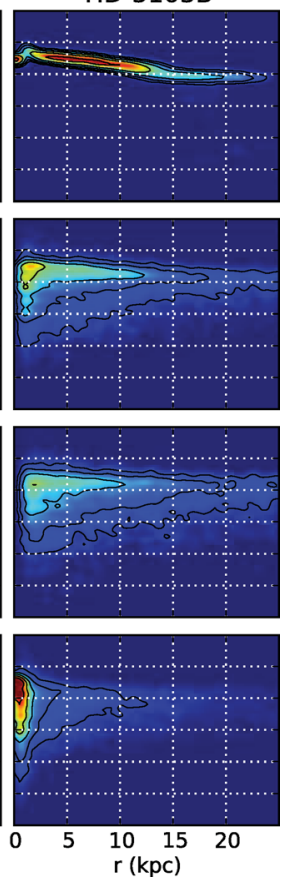

Figure 14. Metallicity radial distributions for gas, thin disc, thick disc and spheroid (from top to bottom).

would have produced more complex abundance distributions. In external regions, however, steeper gradients are found, with metalpoor stars at high radial distances (e.g. Yong, Carney \& Teixera de Almeida 2005). The existence of such a radial profile can indicate that the unobserved inner stellar thin disc may be significantly more metallic than the solar neighbourhood.

Observations of the Milky Way reveal that the different galactic components have characteristic abundance patterns. Navarro et al. (2011) show distinctive abundances for the thin and the thick disc in the solar neighbourhood, with the thick disc being significantly less enriched. The spheroid is known to have a gradient with the outer (halo) parts being very metal poor (Ivezić et al. 2008), and the inner bulge having metallicities around the solar value (Zoccali et al. 2008).

In Fig. 14 we depict the metallicity versus radius distributions for each component identified by the 'kk-means' method in our simulated galaxies. In addition, the first row represents the gas component, with computed gradients ranging from $-0.060 \mathrm{dex}$ $\mathrm{kpc}^{-1}$ in the object HD-5004A to $-0.039 \mathrm{dex} \mathrm{kpc}^{-1}$ in LD-5101A which is the less enriched galaxy.

At first glance, one can appreciate that the more 'discy' a component is, the lower is its dispersion in metallicity for any given radius, with the spheroids showing the highest range of variation in metallicity, and the thin discs the lowest. The thick discs appear here as an intermediate component, with almost the same radial extent as the thin disc but a higher dispersion in their metallicity values. The least massive objects (LD-5003A and LD-5101A) show the lowest metallicity gradient for the thin disc component, whereas a more massive galaxy (HD-5004A and its counterpart HD-5004B) shows a larger gradient, with the run having the least effective set of SF parameters (HD-5004B) having a larger gradient.

Given that our sample is very small, no conclusions can be drawn with respect to the relation between morphological type (or $D / T$ ratio) and metallicity gradient, although there is a similar effect known from observations (Edmunds \& Roy 1993). The difference between HD-5004A and HD-5004B, given that it is the same object with a different set of SF parameters, is however more telling. A reduction in the overall SF efficiency leads to a steeper gradient. Given that the metallicity values in the inner part of both objects are similar, the difference must be due to a reduction in the SF efficiency in the outer parts of the disc, where the density threshold criterion is not always met by the HD-5004B simulation. This effect is present in observations of nearby spirals (Leroy et al. 2008; Bigiel et al. 2010), where a decreasing SF efficiency with density (or galactocentric radius) is found, leading to extremely inefficient SF in the outskirts of discs.

The spheroids show a rather complex structure in Fig. 14. For most objects several bumps in the MDF of the bulge are apparent (see also Fig. 13). As was explained in the preceding section, this is likely the outcome of two different processes driving the formation of the bulge, with the highest metallicity peak being associated with a pseudo-bulge and the lower metallicity distribution being associated with the classical bulge. Further results on the bulge abundances and their formation process will be presented in Obreja et al. (submitted).

Object HD-5103A, and its counterpart HD-5103B, deserves a special mention. Since it has undergone a recent merger, its stellar content is mixed, and the distributions are generally broader for any given radius, with many particles shifting orbit and therefore lower particle densities attained in the metallicity versus radius plane.

\section{$7.3 \alpha$-elements}

A number of works in the literature, using different stellar samples, have studied the abundance of $\alpha$-elements in the bulge, thin disc and thick disc of the Milky Way (e.g. Bensby et al. 2003, 2005; Reddy et al. 2006; Jablonka, Gorgas \& Goudfrooij 2007; Meléndez et al. 2008; Ruchti et al. 2010). According to these works, the Galactic 


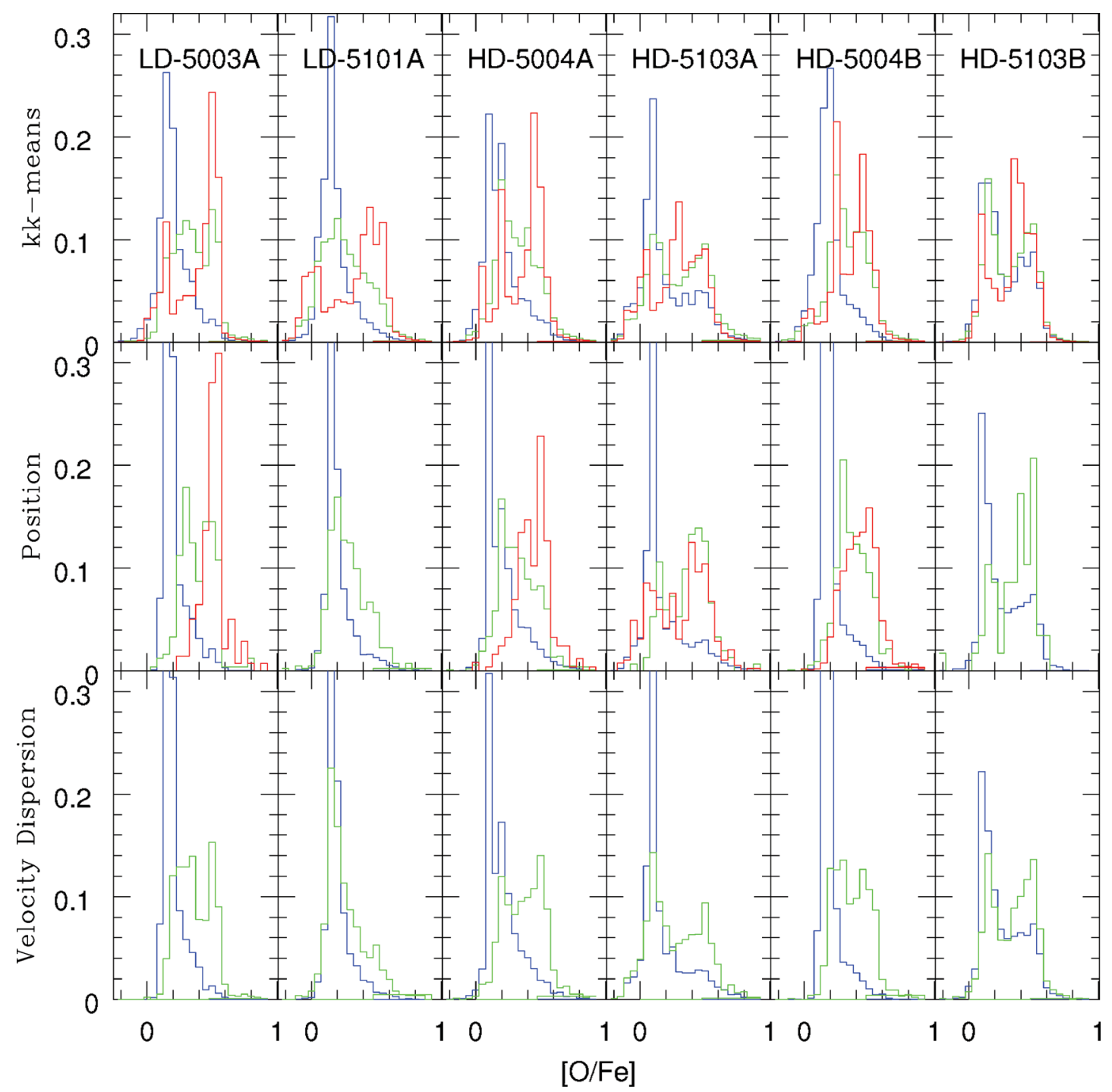

Figure 15. Overabundance distribution of stars in each galaxy component measured as the oxygen ratio over iron.

bulge and thick disc are on average more enhanced in $\alpha$-elements than the thin disc, suggesting a short formation time-scale for these two components. The $\alpha$-elements are in fact mainly produced during Type II SNe on a short time-scale, whereas iron is also produced by Type Ia SNe on a much longer time-scale. An enhanced abundance in $\alpha$-elements can be then interpreted as a signature of a short period of stellar formation, where $\mathrm{SNe}$ Ia have no enough time to dilute the $\alpha$-elements produced by Type II SNe.

Moreover, in the thin and thick discs the abundance of $\alpha$-elements shows tight and distinct trends with metallicity. In the thin disc, this trend has a gentle slope, whereas for the thick disc, some authors (Bensby et al. 2003, 2005) have pointed out the presence of a downturn (or 'knee'). According to these authors, for metallicity values $[\mathrm{Fe} / \mathrm{H}] \lesssim-0.4$, stars in the thick disc have $[\alpha / \mathrm{Fe}]$ values higher than in the thin disc. At $[\mathrm{Fe} / \mathrm{H}] \sim-0.4$, the $[\alpha / \mathrm{Fe}]$ trend has a down-turn, so that it declines towards solar values merging with the thin disc trend for $[\mathrm{Fe} / \mathrm{H}] \gtrsim-0.4$. In contrast, other authors (e.g. Reddy et al. 2006) doubt the existence of this 'knee', but agree in the different behaviour of thin and thick disc stars.
In Fig. 15, we show the distribution of the abundance of $\alpha$-elements represented by oxygen. ${ }^{7}$ In general, all samples of thick disc stars distribute around a median value $(\sim 0.3)$ slightly higher than in the thin disc. For the location-based samples of stars in the spheroid, we also find $\alpha$-enhanced abundances, with median [O/Fe] values $(\sim 0.45)$ higher than in the thick disc. The spheroid samples identified by 'kk-means' have instead a bimodal distribution: there exists a population of stars poor in $\alpha$-elements, as well as another population enhanced in these elements. Such a bimodality could be due to the combined action of two bulge formation mechanisms (classical bulge and pseudo-bulge), and also to different origin of stars in the halo as recently pointed out by Nissen \& Schuster (2010). They suggest that low- $\alpha$ stars in the Galactic halo probably were accreted from dwarf galaxies that had lower SF rates, while the high- $\alpha$ population is formed by the first stars likely originated in a

\footnotetext{
${ }^{7}$ Similar trends are found when the abundance of $\alpha$-elements is represented by magnesium.
} 


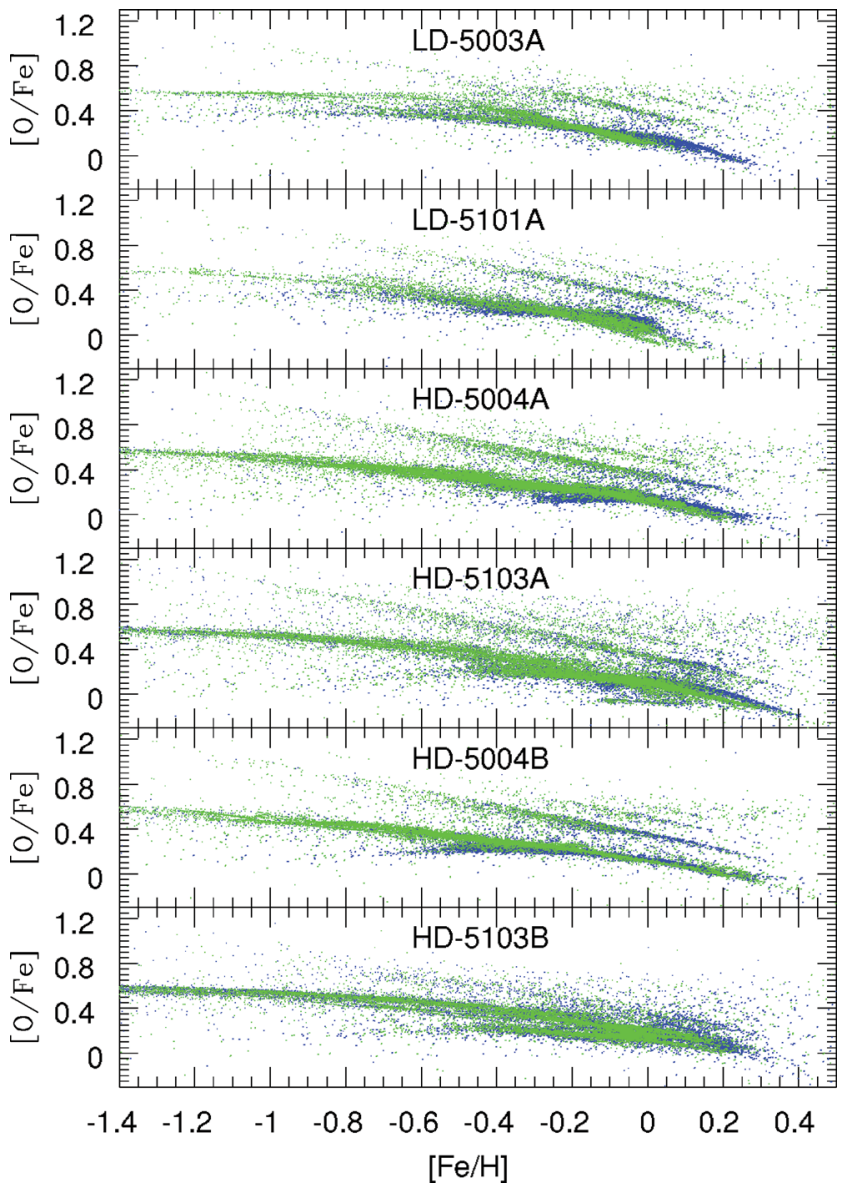

Figure 16. $[\mathrm{O} / \mathrm{Fe}]$ versus $[\mathrm{Fe} / \mathrm{H}]$ ratios for $\mathrm{F}$ and $\mathrm{G}$ stars in the thin and thick discs (blue and green dots, respectively) of each simulated galaxy.

dissipative collapse of the proto-Galactic gas cloud, or by ancient disc or bulge stars heated to the halo by merging processes.

In Fig. 16 we depict $[\mathrm{O} / \mathrm{Fe}]$ versus $[\mathrm{Fe} / \mathrm{H}]$ for disc stars in each simulated galaxy. To compare our results with the observational trends reported by Bensby et al. (2003, 2005), the number of points in Fig. 16 is proportional to the fraction of $\mathrm{F}$ and $\mathrm{G}$ stars in each star particle, computed from its age and metallicity assuming a Salpeter IMF. From this figure we can see that at low metallicities the thick disc stars are generally more $\alpha$-enhanced than those with the same metallicity in the thin disc. This is particularly clear in objects LD5003A and LD-5101A, located in sparsely populated regions of the universe. In these objects, a 'knee' is found at roughly the same metallicity value $([\mathrm{Fe} / \mathrm{H}] \sim-0.4)$ as in the Milky Way observations of Bensby et al. (2003, 2005). We also find for these objects that there are some old thin disc stars overabundant in oxygen. Although less clearly, such an $\alpha$-enhancement of the thick disc low-metallicity stars seems to be also present in the objects HD-5004A, HD-5103A and their counterparts, populating denser environments. We also note that, except for HD-5103B, the plot of $[\mathrm{O} / \mathrm{Fe}]$ versus $[\mathrm{Fe} / \mathrm{H}]$ has secondary branches containing stars of both disc components with high $\alpha$-abundance and metallicity. These secondary curves are mainly populated by young thin disc stars formed in an enriched medium and by old thick disc stars which formed during a rapid episode. These old stars enriched the medium with $\alpha$-elements and then they have been evolving at the same time that the iron abundance has been increasing.

\section{SUMMARY AND CONCLUSIONS}

We have presented a detailed analysis of the photometric and chemical properties of seven simulations of disc galaxies in the $\Lambda \mathrm{CDM}$ scenario. Each simulation is a cosmological zoom-in that includes high-resolution gas and DM particles for the converging region that generates the main object. SF is modelled through a KennincuttSchmidt-like law with a SF efficiency that implicitly accounts for energy feedback. Although the addition of some explicit form of kinetic feedback could presumably lead to simulated galaxy populations in even better agreement with observations (Kereš et al. 2009), in this paper we have preferred to test the minimal conditions for the formation of realistic discs, thereby facilitating the physical interpretation of our results. The methods for chemical feedback and abundance-dependent cooling are also included. The simulations presented in this paper correspond to four different objects selected from both sparsely populated and crowded regions. In order to test the robustness of our results, three of the seven runs consisted of variants with different SF efficiency or resolution.

From the bulge-disc decomposition of their luminosity profiles, we find that our simulated galaxies are disc dominated, with bulge sizes comparable to those observed in late-type spirals. In addition they have also realistic kinematic properties and lie in the region defined by the observational Tully-Fisher relation.

In order to analyse the fine structure of these objects, we have performed a dynamical decomposition of the galactic components based on the total binding energy and the axial and radial components of the angular momentum, normalized with the angular momentum expected for a particle in a circular orbit. We used an unsupervised clustering algorithm which classifies stars by minimizing the sum of the distances of each particle to its cluster centre in the space defined by these three parameters. In this way, we can identify stars from the thin and thick discs, and also from the spheroid, which includes bulge and halo. We will now discuss our main results for each component: spheroid, thin disc and thick disc.

We find that the spheroidal component of the simulated galaxies is composed mainly by old stars, with just a small contribution of stars younger than 5 Gyr. The bulk of stars formed in a rapid collapse at very early times. Afterwards, the SF turned off, and only residual SF is observed at low redshifts. The age and colour profiles for this component show that the stellar content is distributed quite uniformly, as observed in real galaxies, with mean ages almost constant along the galaxy. The only exception is the simulated object HD-5103A and its less efficient SF counterpart HD-5103B where, as a consequence of a recent major merger, an important SF episode took place at redshift $\sim 0.3$. New stars were born in this event, and populated mainly the spheroid, which shows a U-shaped colour profile and a mean age profile younger than the other galaxies. The distribution of metallicity and $\alpha$-elements for stars in the spheroid shows a clear bimodality. Such a bimodality seems to be connected with the different origin of stars in this component. The low-metallicity peak could be related to the classical bulge, while the high-metallicity peak could be associated with a pseudo-bulge formed from instabilities of the inner disc. A detailed analysis of the formation mechanism and time evolution of the bulge component will be presented in a forthcoming paper (Obreja et al. submitted).

The thin disc appears in our simulations as the youngest and most metal-rich component, with roughly solar values for metallicity. The median age of stars ranges from 3.8 to $6.7 \mathrm{Gyr}$, with a just small fraction older than $10 \mathrm{Gyr}$. This is the only component with a 
non-negligible $\mathrm{SF}$ rate $\left(\sim 1 \mathrm{M}_{\odot} \mathrm{yr}^{-1}\right)$ at $z=0$. The radial distribution of ages and colours shows that new stars are forming in the thin disc according to the inside-out paradigm. The U-shape of such profiles indicates (see Martínez-Serrano et al. 2009; Sánchez-Blázquez et al. 2009) that stars have migrated across the galaxy reaching high radial distances.

The thick disc in our simulations contains about 16 per cent of the total stellar mass and has intermediate properties, in between those of the thin disc and the spheroid. From its age and metallicity distributions we find that it is populated by older and more metalpoor stars than in the thin disc, but younger and more metal-rich stars than in the spheroid. The thick disc is not currently forming stars, but its history of SF is quieter than in the spheroid, and extends to a long period of time, with a rate comparable to that observed in the thin disc. The abundance distribution shows that the thick disc is enhanced in $\alpha$-elements, but not as much as that found for the spheroid.

Galaxies located in LD regions of the universe have thick discs where, for low-metallicity stars $([\mathrm{Fe} / \mathrm{H}] \lesssim-0.4)$, the abundance of $\alpha$-elements is clearly enhanced as compared to thin disc stars of the same metallicity. When fitting the vertical density profiles of RGB stars, we find that objects in LD regions have thick disc scale heights $z_{\mathrm{h}}$ that do not change significantly along the disc. According to some authors (Ibata et al. 2009), $z_{\mathrm{h}}$ values roughly constant with the radial distance favour the scenario in which the thickening results from internal (non-merger-driven) instabilities in the thin disc. In the case of galaxies located in HD regions of the universe, we find instead a thick disc scale height that changes with the galactocentric distance, as expected in a merger-induced scenario for the formation of this component. For these galaxies, the trend of $[\alpha / \mathrm{Fe}]$ versus $[\mathrm{Fe} / \mathrm{H}]$ for the thin and thick discs also presents a different behaviour for $[\mathrm{Fe} / \mathrm{H}] \lesssim-0.4$. Nevertheless, now the number of stars with very low metallicities in the thin disc is much smaller than that found in the same component of galaxies in LD regions. Consequently, the trend of $[\alpha / \mathrm{Fe}]$ versus $[\mathrm{Fe} / \mathrm{H}]$ for the thin disc of HD galaxies does not extend to very low metallicities. This fact seems to also point out a merger-driven scenario as the main mechanism for the thick disc formation of galaxies in HD regions. Indeed, the lack of thin disc stars with very low metallicities (i.e. very old ages) for such galaxies seems to favour a formation model in which an early thin disc was almost completely disrupted by a violent event giving rise to the thick disc. Afterwards, a new stellar thin disc was formed from the remaining and already metal-enriched gas. In order to discern the role of the various mechanisms of disc thickening a more detailed analysis of the galaxy evolution at different redshifts evolution is needed. Such an analysis will be reported in a future paper.

\section{ACKNOWLEDGMENTS}

FJMS would like to thank Brad Gibson and the Extragalactic Astrophysics group at the Jeremiah Horrocks Institute for their Kind hospitality and useful discussion. We also thank the anonymous referee for their very useful comments and suggestions.

This work was partially supported by the DGES (Spain) through the grants AYA2009-12792-C03-02 and AYA2009-12792C03-03 from the PNAyA, MICINN, Spain. We also thank the ASTROMADRID network (CAM S2009/ESP-1496) from the Madrid regional government, and the 'Supercomputación y e-Ciencia' Consolider-Ingenio 2010 project (CSD2007-0050), MICINN, Spain. The Centro de Computación Cientifíca (UAM, Spain) has provided computing facilities.

\section{REFERENCES}

Abadi M. G., Navarro J. F., Steinmetz M., Eke V. R., 2003a, ApJ, 591, 499 Abadi M. G., Navarro J. F., Steinmetz M., Eke V. R., 2003b, ApJ, 597, 21 Agertz O., Teyssier R., Moore B., 2011, MNRAS, 410, 1391

Bakos J., Trujillo I., Pohlen M., 2008, ApJ, 683, L103

Barber C. B., Dobkin D. P., Huhdanpaa H., 1996, ACM Trans. Math. Softw., 22, 469

Bensby T., Feltzing S., Lundström I., 2003, A\&A, 410, 527

Bensby T., Feltzing S., Lundström I., Ilyin I., 2005, A\&A, 433, 185

Bigiel F., Leroy A., Walter F., Blitz L., Brinks E., de Blok W. J. G., Madore B., 2010, AJ, 140, 1194

Bournaud F., Elmegreen B. G., Martig M., 2009, ApJ, 707, L1

Brook C. B., Kawata D., Gibson B. K., Freeman K. C., 2004, ApJ, 612, 894

Brook C. B., Gibson B. K., Martel H., Kawata D., 2005, ApJ, 630, 298

Brooks A. M., Governato F., Quinn T., Brook C. B., Wadsley J., 2009, ApJ, 694, 396

Bruzual G., Charlot S., 2003, MNRAS, 344, 1000

Burkert A., 2009, preprint (arXiv:0908.1409)

Carollo D. et al., 2010, ApJ, 712, 692

Catinella B., Giovanelli R., Haynes M. P., 2006, ApJ, 640, 751

Collins M. L. M. et al., 2011, MNRAS, 413, 1548

Croft R. A. C., Di Matteo T., Springel V., Hernquist L., 2009, MNRAS, 400, 43

Dalcanton J. J., Bernstein R. A., 2002, AJ, 124, 1328

Dekel A., Birnboim Y., 2006, MNRAS, 368, 2

Dhillon I., Guan Y., Kulis B., 2004, A Unified View of Kernel k-means, Spectral Clustering and Graph Cuts. Tech. Rep. TR-04-25, University of Texas at Austin, Department of Computer Sciences, Austin, TX

Domínguez-Tenreiro R., Tissera P. B., Sáiz A., 1998, ApJ, 508, L123

Edmunds M. G., Roy J.-R., 1993, MNRAS, 261, L17

Fall S. M., 1983, in Athanassoula E., ed., Proc. IAU Symp. 100, Internal Kinematics and Dynamics of Galaxies. Kluwer, Dordrecht, p. 391

Fall S. M., Efstathiou G., 1980, MNRAS, 193, 189

Fuhrmann K., 1998, A\&A, 338, 161

Gadotti D. A., 2008, MNRAS, 384, 420

Garnett D. R., Shields G. A., Skillman E. D., Sagan S. P., Dufour R. J., 1997, ApJ, 489, 63

Gavilán M., Buell J. F., Mollá M., 2005, A\&A, 432, 861

Gilmore G., Reid N., 1983, MNRAS, 202, 1025

Gilmore G., Wyse R. F. G., Jones J. B., 1995, AJ, 109, 1095

Gonzalez-Perez V., Castander F. J., Kauffmann G., 2011, MNRAS, 411, 1151

Governato F., Willman B., Mayer L., Brooks A., Stinson G., Valenzuela O., Wadsley J., Quinn T., 2007, MNRAS, 374, 1479

Governato F. et al., 2009, MNRAS, 398, 312

Graham A. W., Worley C. C., 2008, MNRAS, 388, 1708

Grevesse N., Sauval A. J., 1998, Space Sci. Rev., 85, 161

Guedes J., Callegari S., Madau P., Mayer L., 2011, ApJ, 742, 76

Ibata R., Mouhcine M., Rejkuba M., 2009, MNRAS, 395, 126

Ivezić Ž. et al., 2008, ApJ, 684, 287

Iwamoto K., Brachwitz F., Nomoto K., Kishimoto N., Umeda H., Hix W. R., Thielemann F.-K., 1999, ApJS, 125, 439

Jablonka P., Gorgas J., Goudfrooij P., 2007, A\&A, 474, 763

Jurić M. et al., 2008, ApJ, 673, 864

Karatzoglou A., Smola A., Hornik K., 2004, J. Statistical Software, 11, 1

Kereš D., Katz N., Weinberg D. H., Davé R., 2005, MNRAS, 363, 2

Kereš D., Katz N., Davé R., Fardal M., Weinberg D. H., 2009, MNRAS, 396, 2332

Kormendy J., Kennicutt R. C., Jr, 2004, ARA\&A, 42, 603

Kravtsov A. V., 2003, ApJ, 590, L1

Laurikainen E., Salo H., Buta R., Knapen J. H., 2007, MNRAS, 381, 401

Leroy A. K., Walter F., Brinks E., Bigiel F., de Blok W. J. G., Madore B., Thornley M. D., 2008, AJ, 136, 2782

Luck R. E., Kovtyukh V. V., Andrievsky S. M., 2006, AJ, 132, 902

MacArthur L. A., González J. J., Courteau S., 2009, MNRAS, 395, 28

Martínez-Serrano F. J., Serna A., Domínguez-Tenreiro R., Mollá M., 2008, MNRAS, 388, 39 
Martínez-Serrano F. J., Serna A., Doménech-Moral M., DomínguezTenreiro R., 2009, ApJ, 705, L133

Meléndez J. et al., 2008, A\&A, 484, L21

Navarro J. F., 1998, preprint (astro-ph/9807084)

Navarro J. F., White S. D. M., 1994, MNRAS, 267, 401

Navarro J. F., Abadi M. G., Venn K. A., Freeman K. C., Anguiano B., 2011 , MNRAS, 412, 1203

Nissen P. E., Schuster W. J., 2010, A\&A, 511, L10

Obreja A., Domínguez-Tenreiro R., Martínez-Serrano F., Doménech-Moral M., Serna A., 2012, MNRAS, submitted

Oohama N., Okamura S., Fukugita M., Yasuda N., Nakamura O., 2009, ApJ, 705,245

Pancino E., Carrera R., Rossetti E., Gallart C., 2010, A\&A, 511, A56

Peng C. Y., Ho L. C., Impey C. D., Rix H.-W., 2010, AJ, 139, 2097

Power C., Knebe A., 2006, MNRAS, 370, 691

Prunet S., Pichon C., Aubert D., Pogosyan D., Teyssier R., Gottloeber S., 2008, ApJS, 178, 179

Quinn P. J., Hernquist L., Fullagar D. P., 1993, ApJ, 403, 74

Reddy B. E., Lambert D. L., Allende Prieto C., 2006, MNRAS, 367, 1329

Robin A. C., Reylé C., Derrière S., Picaud S., 2003, A\&A, 409, 523

Roškar R., Debattista V. P., Quinn T. R., Stinson G. S., Wadsley J., 2008, ApJ, 684, L79

Ruchti G. R. et al., 2010, ApJ, 721, L92

Ruiz-Lapuente P., Blinnikov S., Canal R., Mendez J., Sorokina E., Visco A., Walton N., 2000, Mem. Soc. Astron. Ital., 71, 435

Sáiz A., Domínguez-Tenreiro R., Tissera P. B., Courteau S., 2001, MNRAS, 325,119

Sales L. V. et al., 2009, MNRAS, 400, L61

Salpeter E. E., 1955, ApJ, 121, 161

Sánchez-Blázquez P., Courty S., Gibson B. K., Brook C. B., 2009, MNRAS, 398, 591

Scannapieco C., Tissera P. B., 2003, MNRAS, 338, 880

Scannapieco C., Tissera P. B., White S. D. M., Springel V., 2008, MNRAS, 389,1137

Scannapieco C., White S. D. M., Springel V., Tissera P. B., 2009, MNRAS, 396, 696

Scannapieco C., Gadotti D. A., Jonsson P., White S. D. M., 2010, MNRAS, 407, L41

Scannapieco C. et al., 2011a, preprint (arXiv:1112.0315)

Scannapieco C., White S. D. M., Springel V., Tissera P. B., 2011b, MNRAS, 417,154
Schölkopf B., Smola A., Müller K.-R., 1998, Neural Comput., 10, 1299

Schönrich R., Binney J., 2009a, MNRAS, 396, 203

Schönrich R., Binney J., 2009b, MNRAS, 399, 1145

Schuster W. J., Moitinho A., Márquez A., Parrao L., Covarrubias E., 2006, A\&A, 445, 939

Serna A., Domínguez-Tenreiro R., Sáiz A., 2003, ApJ, 597, 878

Seth A. C., Dalcanton J. J., de Jong R. S., 2005, AJ, 130, 1574

Shaver P. A., McGee R. X., Newton L. M., Danks A. C., Pottasch S. R., 1983, MNRAS, 204, 53

Shen S., Mo H. J., White S. D. M., Blanton M. R., Kauffmann G., Voges W., Brinkmann J., Csabai I., 2003, MNRAS, 343, 978

Siegel M. H., Majewski S. R., Reid I. N., Thompson I. B., 2002, ApJ, 578, 151

Sommer-Larsen J., Götz M., Portinari L., 2003, ApJ, 596, 47

Soubiran C., Bienaymé O., Siebert A., 2003, A\&A, 398, 141

Springob C. M., Masters K. L., Haynes M. P., Giovanelli R., Marinoni C., 2007, ApJS, 172, 599

Stinson G. S., Bailin J., Couchman H., Wadsley J., Shen S., Nickerson S., Brook C., Quinn T., 2010, MNRAS, 408, 812

Talbot R. J., Jr, Arnett W. D., 1973, ApJ, 186, 51

Torrey P., Vogelsberger M., Sijacki D., Springel V., Hernquist L., 2011, preprint (arXiv: 1110.5635)

van Zee L., Salzer J. J., Haynes M. P., O’Donoghue A. A., Balonek T. J., 1998, AJ, 116, 2805

Villalobos Á., Helmi A., 2008, MNRAS, 391, 1806

Wada K., Norman C. A., 2007, ApJ, 660, 276

Walker I. R., Mihos J. C., Hernquist L., 1996, ApJ, 460, 121

Weinzirl T., Jogee S., Khochfar S., Burkert A., Kormendy J., 2009, ApJ, 696, 411

White S. D. M., 1984, ApJ, 286, 38

Woosley S. E., Weaver T. A., 1995, ApJS, 101, 181

Yoachim P., Dalcanton J. J., 2005, ApJ, 624, 701

Yoachim P., Dalcanton J. J., 2006, AJ, 131, 226

Yoachim P., Dalcanton J. J., 2008, ApJ, 683, 707

Yong D., Carney B. W., Teixera de Almeida M. L., 2005, AJ, 130, 597

Zaritsky D., Kennicutt R. C., Jr, Huchra J. P., 1994, ApJ, 420, 87

Zoccali M., Hill V., Lecureur A., Barbuy B., Renzini A., Minniti D., Gómez A., Ortolani S., 2008, A\&A, 486, 177

This paper has been typeset from a $\mathrm{T}_{\mathrm{E}} \mathrm{X} / \mathrm{L} \mathrm{T}_{\mathrm{E}} \mathrm{X}$ file prepared by the author. 